\title{
LA VÍA ECLÉCTICA DE PRODUCCIÓN Y CONSUMO DE DATOS ESPACIALES
}

\author{
Jorge DEL RÍO SAN JOSÉ (riosanjo@jcyl.es) \\ Junta de Castilla y León. Delegación Territorial de Valladolid. Servicio Terri- \\ torial de Medio Ambiente
}

Recibido: $29 / 03 / 2015$

Aceptado: 29/09/2015

RESUMEN: Los datos espaciales han irrumpido con fuerza en las agendas de gran parte de la sociedad lo que ha provocado la aparición de numerosos fenómenos, controversias y la aplicación de nuevos y variados paradigmas que intentan adaptar la producción a los nuevos patrones de consumo de información.

La vía ecléctica, en la que conviven los distintos paradigmas, es el hilo conductor propuesto en este artículo de revisión para describir los cambios actuales en la creación, gestión y uso de los datos espaciales. Para ello se clasifican e inventarían las causas y efectos de las prácticas de producción y consumo que acontecen en las geocomunidades. También se cartografía el mapa de la ciencia (2003-2014) en materia de neogeografía, voluntariado de la información geográfica y sociedad capacitada geoespacialmente.

La revisión concluye que se ha observado un gran interés en el fenómeno neogeográfico sin extender el análisis al conjunto del fenómeno de producción y consumo de datos espaciales. La vía ecléctica propuesta en esta revisión detecta áreas de trabajo y estudio para la comunidad científica, e identifica aspectos claves en la creación y gestión de datos y geocomunidades.

$\boldsymbol{P A L A B R A S ~ C L A V E}$ : Revisión, Google Académico, neogeografía, voluntariado de información geográfica, sociedad capacitada geoespacialmente, infraestructura de datos espaciales, sistemas de información geográfica de participación pública, contenido generado por el usuario, informacionalismo, cadena de suministro, lean, agile, cloud, punto de desacoplamiento, determinismo, constructivismo.

THE ECLECTIC WAY OF PRODUCTION AND CONSUMPTION OF SPATIAL DATA

ABSTRACT: Spatial data have burst onto the agendas of society, which has resulted in the current context the emergence of numerous events, controversies and application of new and varied paradigms trying to adapt production to the new patterns of consumption of information.

The eclectic way, in the live different paradigms, is the theme proposed in this review article describing the current changes in the creation, management and use of spatial data. This paper classifies and enumerates the causes and effects of the strategies and practices of production and consumption that occur in the geo- communities, whereupon it draws science map (20032014) on neogeography, volunteer geographic information and spatially enabled society.

This review concludes that there has been a great interest in neogeography, without extending the analysis to the whole phenomenon of production y consumption of spatial data. The eclectic 
approach proposed in this review detects areas of work for the scientific community and identifies key aspects in the creation and management of data and geo-communities.

KEY WORDS: Review Google scholar, neogeography, VGI, spatially enabled society, SDI, PPGIS, UGC, informationalism, supply chain paradigm, lean, agile, cloud, green, decoupling point, determinism, constructivism.

\section{EL ATASCO ACTUAL}

En los últimos años hemos asistido a un meme: «los profundos cambios en torno a los mapas, la cartografía, los datos espaciales y la geografía». Los medios de comunicación de los ámbitos científico, técnico, de masas, o de la larga cola de Internet, se hacen eco de forma más o menos recurrente de la aparición de fenómenos novedosos en los que la componente geográfica es la protagonista (CAPEL, 2012).

Los datos espaciales han entrado en las agendas de gran parte de la sociedad, (VIRRANTAUS ET AL, 2009) pero ¿por qué motivo?, ¿qué es lo qué ha cambiado realmente?, ¿tiene algo en común este tren de noticias? Este artículo plantea una respuesta a partir de la revisión de la literatura reciente sobre el tema: el cambio de mayor relevancia se está produciendo en los modos de producción y consumo de información geográfica.

El ritmo de la transformación está siendo rápido e intenso si lo comparamos con otros momentos que la historia de la ciencia y la tecnología nos han mostrado, tanto por lo concentrado en el tiempo, como por la severidad de su impacto. El análisis de este fenómeno ha provocado la aparición de controversias, entre ellas, la neogeografía y el voluntariado de la información geográfica son las más visibles aunque no son las únicas (ELWOOD y LESZCZYNSKI, 2011).

¿Dónde nos hemos atascado en el análisis de la situación actual? Utilicemos los mapas como caso de estudio, ya que han sido el canal de mayor éxito en el registro y difusión de los datos espaciales. Así, en el ámbito cartográfico DODGE et al. (2009) plantean como causa de este atasco la insuficiencia metodológica para abordar la investigación de los actuales modos de cartografiar. Estos autores afirman que la historia de la cartografía se ha basado en un discurso lineal de progreso y perfeccionamiento que parece insuficiente para enfrentar el estudio del presente. Un presente donde EDNEY (2011) nos indica que existe una cartografía sin progreso y una superposición de modos cartográficos que parecen competir, y en el que existe una mezcla de roles de producción y consumo en lo que se ha denominado prosumidores (RITZER y JUNGERSON, 2010). A modo de ejemplo podemos reseñar la proliferación de mapas persuasivos que escapan de las normas de visualización científica y 
funcionan mediante diferentes estilos retóricos de comunicación (MUEHLENHAUS, 2013, 2014).

La huida o el rechazo de las posturas positivistas o neopositivistas no son nuevas en la historia de la cartografía. HARLEY (1990) definió el mapa como un constructo social cuya interpretación debe ser deconstruida en tres contextos: el del cartógrafo, el de otros mapas y el de la sociedad. DODGE et al., (2009) nos plantean si los enfoques ofrecidos por la sociología de la ciencia y tecnología pueden aportar una vía interesante, y nos sugieren una posición epistemológica pragmática que incorpore simultáneos enfoques teóricos como medio para ayudarnos a repensar los mapas y abrir la caja negra de la tecnología. El reto, la oportunidad y la provocación, están lanzados en este debate. ¿Cuál es el modo actual de producción y consumo de los datos espaciales?

\section{EL ACERVO}

La historia ha prestado mayor atención al contenido de los mapas, a su diseño y a la producción cartográfica que al consumo (EDNEY, 2005). No es el objetivo de este texto desarrollar una historiografía de los modos de producción y consumo de datos espaciales, sino ofrecer algunas pinceladas que nos muestren parte del capital cultural que hemos heredado y que hoy en día siguen vigentes (CUADRO 1).

Cuadro 1. Los modos históricos de producción y consumo de mapas

\begin{tabular}{llll}
\hline Modo histórico producción & \multicolumn{1}{c}{ Valor } & \multicolumn{1}{c}{ Acervo } & \multicolumn{1}{c}{ Consumo } \\
\hline Manual & Uso & Función del mapa & Persona, tribu \\
\hline Artesanal & Cambio & $\begin{array}{l}\text { Especialización de la produc- } \\
\text { ción }\end{array}$ & Monopsonio \\
\hline Talleres & $\begin{array}{l}\text { Signo y sím- } \\
\text { bolo }\end{array}$ & $\begin{array}{l}\text { Diversificación y diferencia- } \\
\text { ción de la producción: marca } \\
\text { y calidad }\end{array}$ & $\begin{array}{l}\text { Oligopolio y Oli- } \\
\text { gopsonio }\end{array}$ \\
\hline Casas comerciales-Gremial & $\begin{array}{l}\text { Amortiza- } \\
\text { ción del va- } \\
\text { lor de uso }\end{array}$ & $\begin{array}{l}\text { Creación de geocomunidades } \\
\text { y activo empresarial }\end{array}$ & $\begin{array}{l}\text { Organizaciones } \\
\text { mercantiles }\end{array}$ \\
\hline Producción en masa & $\begin{array}{l}\text { Objeto de Profesionalización e institu- } \\
\text { consumo }\end{array}$ & $\begin{array}{l}\text { Consumo de ma- } \\
\text { cionalización }\end{array}$ \\
\hline Informacionalismo & $\begin{array}{l}\text { Objeto de } \\
\text { consumo vir- Virtualización y prosumido- } \\
\text { tual: Infor- } \\
\text { mación }\end{array}$ & $\begin{array}{l}\text { Personalización } \\
\text { de producción y } \\
\text { consumo }\end{array}$ \\
\hline
\end{tabular}

FUENTE: Elaboración del autor

\section{II.1. De valor de uso a objeto de consumo}

La posición, las rutas o el espacio, son los elementos geográficos que más han interesado al ser humano. Un ejemplo temprano lo encontramos en el 
mapa hallado en Navarra de hace 13.660 años $^{1}$ (UTRILLA et al., 2009) o en el petroglifo de Bedolina (2000-1500 a.C.). Este interés por los datos espaciales impregnó rápidamente de valor de uso al dato espacial.

A lo largo de las primeras sociedades, civilizaciones clásicas y del medievo, el patrón no ha variado de manera sustancial. La producción se monopolizó en roles de «eruditos» y «copistas», mientras que el consumo del dato, ahora registrado en mapas, era un monopsonio restringido a las élites de poder de cada sociedad. El dato espacial se fue perfeccionado y los usos del mapa fueron adaptándose al contexto social. Apareció un incipiente valor de cambio asociado al mapa, donde se valoraban relaciones de intercambio entre productores y consumidores.

La ruptura del patrón de consumo y producción artesanal se originó y consolidó en Europa a comienzos del siglo XVI. La aparición de la imprenta abarató los costes de acceso a la información; los descubrimientos transoceánicos proporcionaron nuevos datos geográficos y el impulso de las universidades desarrolló modelos de proyección que permitían registrar esa información en mapas. La producción de los «nuevos mapas» se concentró en un nuevo oligopolio: los talleres. La producción de los talleres facilitó la accesibilidad a nuevos actores deseosos de mapas y como consecuencia de su incorporación, la agencia del mapa se amplió. En el año 1500 el número de mapas disponibles era de uno por cada 1400 habitantes; en el año 1600 esta cifra se incrementó hasta alcanzar el valor de un mapa por cada 7,3 habitantes (KARROW, 2007).

Entre los nuevos sujetos de consumo destacó una incipiente burguesía, pujante, curiosa y ávida de estatus, que impulsó la demanda. El mapa se integró en el capital cultural de esta nueva clase económica, que añadió al consumo de mapas un significado social de prestigio. El mapa se había convertido en un objeto cuyo consumo no era cuestión de necesidad sino de signo. Al valor del mapa se le otorgó un precio.

Este modo de producción y consumo de datos espaciales no fue el único que vio la luz en este periodo. Otro grupo social relevante apareció en escena: el formado por las primeras corporaciones mercantiles. Las «casas» crearon sistemas de producción y actualización cartográfica que guardan analogías con las actuales infraestructuras de datos espaciales $(S D I)$. El dato espacial pasó a ser un activo empresarial, un secreto comercial, y su consumo implicó la

\footnotetext{
${ }^{1}$ Este mapa almacena información ambiental y espacial vinculada a la necesidad de tomar decisiones sobre aspectos relevantes de la vida cotidiana del momento, como la caza (CLARKE, 2013).
} 
aparición de una clara amortización del valor de uso como parte esencial de la rentabilidad mercantil.

La incipiente producción en serie provocó una cierta equifinalidad, es decir, el mercado se encontró ante una situación en la que distintos mapas representaban el mismo territorio. Este hecho desencadenó la búsqueda de una diferenciación de la producción. La solución se logró mediante dos procesos: por un lado el nacimiento de la imagen de marca y de calidad asociada a los distintos talleres, especialmente lograda a través de la actualización de los datos y del diseño cartográfico; por otro lado, mediante la diversificación del objeto de consumo, lo que propició la creación de nuevos productos disponibles en múltiples formatos, entre los que destacaron por su éxito los atlas. A través de los talleres y los mapas nacieron las primeras relaciones simbólicas entre la marca y el producto cartográfico.

En el periodo comprendido entre la revolución industrial y el comienzo de la última mitad del siglo XX el patrón de producción adquirió matices industriales de creciente profesionalización e institucionalización. En el siglo XVIII se crearon organismos gubernamentales que mediaban la producción cartográfica. A la par, el consumo de datos espaciales fue progresivamente extendiéndose a toda la sociedad y llegó a propiciar un consumo de masas. El acceso a los mapas dejó de estar reservado a un grupo social concreto y se extendió a toda la sociedad, convirtiéndose en parte integrante de los sistemas educativos. Por último, la finalidad del mapa se diversificó, afianzándose como canal de comunicación y recurso científico.

El denominador común de este primer periodo es la dominancia del determinismo de la producción sobre el consumo: es la producción la que empuja al consumo.

\section{II.2. Del mapa al dato espacial}

El cambio más trascendente del último cuarto del siglo XX ha sido la desvinculación del dato espacial del mapa. El producto ha mutado; el dato espacial pasa a ser parte integrante de las bases de datos geoespaciales. A partir de este momento el consumo de los datos espaciales no se realiza exclusivamente en forma de mapas. Estamos ante un nuevo paradigma tecnológico, el informacionalismo (CASTELLS, 2004) en el que cambian los modos de desarrollo y uso de los datos espaciales.

En el siglo actual la implantación de Internet y la difusión de la web 2.0 han sido los últimos saltos en este devenir. La cartografía en Internet se desarrolla casi desde la aparición de la Red. A pesar de su corta historia se han realizado ya compilaciones sobre ella (PLEWE, 2007). Algunas repasan las tecnologías 
facilitadoras (HAKLAY et al., 2008), mientras que otras destacan los efectos sobre la sociedad y la comunidad científica (CAPEL, 2012). Los desarrollos técnicos han provocado un cambio en los modos de producción y consumo que han dado origen al nacimiento de una prolija y novedosa terminología, un mercado lingüistico (BOURDIEU, 2002) que intenta definir y describir una cronología, en ocasiones una taxonomía, o en otras, una simple descripción (HAKLAY et al., 2012).

Desde la perspectiva de la manufactura los datos espaciales se conciben como herramienta que forma parte de la infraestructura tecnológica. Ésta es una visión instrumental que equipara los datos espaciales con el resto de la información, perdiendo por ello su condición singular y son objeto de las mismas tareas que el resto de los datos: captura, control, calidad, análisis o comunicación. Los datos espaciales están por lo tanto subordinados a otros procesos como la toma de decisiones, al seguimiento de las cadenas de producción y distribución o la mejora de la gestión de proceso de negocio (WESKE, 2012).

Desde la perspectiva del consumo, el cambio de mayor relevancia en esta última etapa es el producido por la trasformación del consumidor en un agente activo que es capaz de producir datos, lo cual se ha logrado porque un conjunto de empresas, instituciones y organizaciones han lanzado servicios y aplicaciones en Internet que ponen a disposición de los usuarios los medios para generar datos, mejorarlos, agregarlos, analizarlos, y publicarlos (BERDEOU, 2002). El consumidor puede acceder a los medios de producción y análisis con un coste bajo de aprendizaje, desde múltiples soportes y con escasa complejidad de uso.

Una de las consecuencias indirectas de la conversión del dato espacial en un objeto de consuno virtual, es la posibilidad de cuantificar el interés que despierta en la sociedad. Las cifras actuales de producción y consumo de mapas indexados en Internet ofrecen valores muy llamativos (CUADRO 2). Estos valores nos muestran el interés que despiertan en la sociedad los datos espaciales y los mapas. Este volumen y recubrimiento de datos espaciales sobre el mundo que nos rodea produce lo que ARIZA (2012) ha denominado una cartografia continua, que describe como un fenómeno que va de lo local a lo global, sin costuras, con un continuo de aplicaciones y posibilidades.

De manera simultánea, parte de la producción más corporativa de los datos, la que atañe a la esfera pública y privada, comienza a liberarse mediante licencias de uso, gracias al desarrollo normativo y al principio de datos abiertos en infraestructuras de datos espaciales $(S D I)$. 
El denominador común de este último periodo es que se introduce una construcción social de la producción que convive con el determinismo de la etapa anterior (FIGURA 1). El consumo se convierte en una fuerza motriz que tira de la producción. Para identificar el alcance de este fenómeno se introduce el concepto de punto de desacoplamiento ${ }^{2}$ en los modos de producción como instrumento para medir el efecto «pull» del consumo sobre la producción (HoEKstra y Romme, 1992; VAN HoeK, 1998; OlHager, 2012).

Cuadro 2. La producción y consumo de mapas en Internet

\begin{tabular}{lcccc}
\hline \multicolumn{1}{c}{ Palabras clave } & $\begin{array}{c}\text { «Map» } \\
\text { (Inglés) }\end{array}$ & $\begin{array}{c}\text { «Maps» } \\
\text { (Inglés) }\end{array}$ & $\begin{array}{c}\text { Mapa } \\
\text { (español) }\end{array}$ & $\begin{array}{c}\text { Mapas } \\
\text { (español) }\end{array}$ \\
\hline $\begin{array}{l}\text { Número de contenidos } \\
\text { indexados }\end{array}$ & 5.150 .000 .000 & 1.460 .000 .000 & 1.190 .000 .000 & 198.000 .000 \\
\hline $\begin{array}{l}\text { Número de búsquedas } \\
\text { mensuales }\end{array}$ & 11.100 .000 & 83.100 .000 & 4.090 .000 & 4.090 .000 \\
\hline
\end{tabular}

FUENTE: Elaboración del autor a partir de datos obtenidos de Google (acceso 19/2/2015)

Figura 1. Determinismo y constructivismo en la producción y consumo
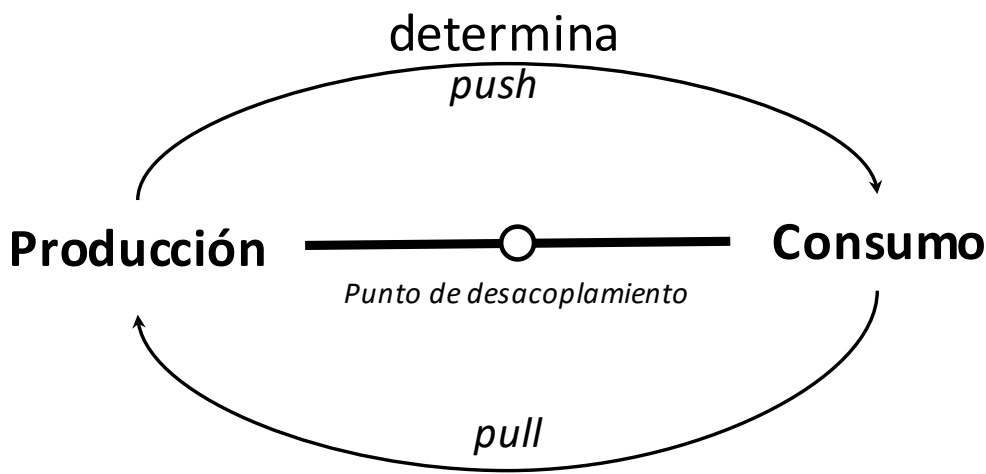

\section{construye}

FUENTE: Elaboración del autor

\footnotetext{
${ }^{2}$ El punto de desacoplamiento de la demanda, también denominado punto de congelación o punto de penetración de un pedido, es el lugar en la cadena de valor donde el producto está vinculado a un pedido específico del cliente. Este punto divide las decisiones productivas realizadas bajo incertidumbre, de las decisiones tomadas en condiciones de certeza, al conocer la demanda. Además sirve a otro propósito: separa el determinismo de la producción, de la construcción social del consumo.
} 


\section{II.3. Los nuevos paradigmas productivos}

De forma paralela al desarrollo del informacionalismo, en la manufactura se produce una proliferación de nuevos paradigmas en la producción de bienes y servicios (CUADRO 3). Estos paradigmas conviven y se superponen en el tiempo. La academia concluye que hay paradigmas productivos que se adaptan mejor a determinadas circunstancias de mercado y económicas (GOLDSBY et al. 2006), a cada tipo de producto (MASON-JONES, 2000), al ciclo de vida del producto (VONDEREMBSE, et al., 2006), o a la disponibilidad de tecnologías de la información (BI, 2014).

El esfuerzo que supone la adopción de cambios productivos provoca la necesidad de construir indicadores para evaluar el grado de desempeño, el efecto y el nivel de adopción de cada paradigma por parte de una organización o de un sector productivo concreto (DUBEY y GUNASEKARAN, 2015). Los indicadores se construyen mediante técnicas multicriterio e índices de agregación que se validan empíricamente (LAU, 2011; O'BRIEN, 2013; AGARWAL et al., 2006).

Desde la esfera de las SDI se realiza un esfuerzo en la evaluación de los datos espaciales. Los indicadores propuestos miden distintos ámbitos, desde el estado actual de desarrollo, el desempeño, el coste eficacia o la utilización de los productos y los servicios, hasta el impacto en la sociedad (MORERA et al., 2012). Sin embargo, no existe literatura que describa los paradigmas de producción y consumo empleados en los datos espaciales ni en el grado de adopción.

Cuadro 3. Los actuales paradigmas en los sistemas productivos

\begin{tabular}{|c|c|c|}
\hline Paradigma & Descripción & Referencia \\
\hline Lean (L) & $\begin{array}{l}\text { Capacidad de mejorar el proceso de valor } \\
\text { agregado mediante la eliminación de } \\
\text { muda. La estrategia consiste en maximizar } \\
\text { el rendimiento y minimizar los costes. }\end{array}$ & $\begin{array}{l}\text { (WOMACK et al., 1990; } \\
\text { HINES et al., 2004; } \\
\text { MELTON, 2005) }\end{array}$ \\
\hline Agile (A) & $\begin{array}{l}\text { Capacidad de adelantarse y/o adaptarse rá- } \\
\text { pidamente y de manera rentable a los cam- } \\
\text { bios impredecibles en los mercados, y al } \\
\text { aumento de los niveles de turbulencia am- } \\
\text { biental tanto en términos de volumen } \\
\text { como de variedad. La estrategia consiste } \\
\text { en satisfacer las necesidades individuales } \\
\text { de los clientes. }\end{array}$ & $\begin{array}{l}\text { CHRISTOPHER, 2000; } \\
\text { CHRISTOPHER } \\
\text { TOWILL, } \\
\text { AGARWAL et al., } \\
\text { 2007; GUNASEKARAN } \\
\text { y YUSUF, 2002.) }\end{array}$ \\
\hline
\end{tabular}




\begin{tabular}{|c|c|c|}
\hline Green $(\mathrm{G})$ & $\begin{array}{l}\text { Capacidad de reducir los riesgos e impac- } \\
\text { tos ambientales al tiempo que mejora la } \\
\text { eficiencia ecológica. La estrategia es au- } \\
\text { mentar los beneficios al reducir los costes } \\
\text { ambientales mediante el ecodiseño y la } \\
\text { evaluación del ciclo de vida del producto. }\end{array}$ & $\begin{array}{ll}\text { (RAO y HOLT, } & 2005 ; \\
\text { LAU, 2011; } & \text { DEIF, } \\
2011) & \end{array}$ \\
\hline Resilience & Capacidad de mantener o recuperar el va- & (CHRISTOPHER \\
\hline $\begin{array}{l}\text { Resistant } \\
\text { (RE) }\end{array}$ & $\begin{array}{l}\text { lor agregado tras una perturbación. Ambos } \\
\text { objetivos deben conseguirse dentro de un } \\
\text { período de tiempo aceptable y con un } \\
\text { coste admisible, reduciendo el daño de la } \\
\text { perturbación mediante la gestión del } \\
\text { riesgo. La estrategia es diferir el fallo. }\end{array}$ & $\begin{array}{l}\text { PECK, 2004; PECK, } \\
\text { 2005) }\end{array}$ \\
\hline $\begin{array}{l}\text { Reconfigu- } \\
\text { rable (RC) }\end{array}$ & $\begin{array}{l}\text { Capacidad de actualizar rápidamente el } \\
\text { sistema mediante la modularidad, inter- } \\
\text { operabilidad, flexibilidad, escalabilidad, } \\
\text { convertibilidad y diagnóstico, para ajustar } \\
\text { la capacidad y funcionalidad de la produc- } \\
\text { ción en respuesta a cambios tecnológicos } \\
\text { y de mercado. La estrategia es lograr ven- } \\
\text { taja competitiva. }\end{array}$ & $\begin{array}{l}\text { (MEHRABI et al., 2000; } \\
\text { ELMARAGHY, 2005) }\end{array}$ \\
\hline Cloud ( C ) & $\begin{array}{l}\text { Capacidad de acceder bajo demanda, de } \\
\text { manera ubicua y en la red, a un conjunto } \\
\text { compartido de recursos de fabricación } \\
\text { configurables que pueden ser rápidamente } \\
\text { aprovisionados y liberados con un es- } \\
\text { fuerzo mínimo de gestión o de interacción } \\
\text { con el proveedor de servicios. La estrate- } \\
\text { gia es la explotación de costes marginales }\end{array}$ & $(\mathrm{XU}, 2012)$ \\
\hline $\begin{array}{ll}\text { Web } & 2.0 . \\
(\mathrm{W}) & \end{array}$ & $\begin{array}{l}\text { Capacidad de crear prosumidores que par- } \\
\text { ticipen en el diseño, fabricación, marke- } \\
\text { ting y esfuerzo de ventas. La estrategia es } \\
\text { liberar los medios de producción. }\end{array}$ & (O'REILLY, 2007) \\
\hline $\begin{array}{l}\text { Sustainable } \\
\text { (S) }\end{array}$ & $\begin{array}{l}\text { Capacidad de las 6Rs: remanufacturar, re- } \\
\text { utilizar, rediseñar, reciclar, recuperar y re- } \\
\text { ducir. La estrategia es maximizar el valor } \\
\text { del producto. }\end{array}$ & (BI, 2011) \\
\hline Mix & $\begin{array}{l}\text { Capacidad de adaptarse a variedad mercado } \\
\text { cambios mediante la introducción de parac } \\
\text { gran a varios de los anteriores. } \\
\text { LeanAgile (NAYLOR et al., 1999) } \\
\text { CloudAgile (MACIÁ PÉREZ et al. 2012) } \\
\text { LARG lean, agile, resilient, and green } \\
\text { MACHADO, 2011) }\end{array}$ & $\begin{array}{l}\text { y a la velocidad de los } \\
\text { gmas mixtos que inte- } \\
\text { CARVALHO y CRUZ- }\end{array}$ \\
\hline
\end{tabular}

FUENTE: Elaboración propia 


\section{EL MÉTOdO DE ANÁliSIS: EL PROCESO ANALÍtico JERÁRQUICO}

Disponer de un modelo que describa la situación actual de la producción y el consumo de datos espaciales es útil para orientar la gestión, el liderazgo y la organización de las geocomunidades. Para elaborar este modelo se utiliza el proceso analítico jerárquico AHP (SAATY, 1980) que requiere de la construcción de árboles de jerarquía de los criterios de evaluación y de un conjunto de alternativas.

En los apartados siguientes, del IV al VIII, se recopilan los principales fenómenos sobre la geoesfera obtenidos en la revisión de la literatura reciente. A partir de ellos se construyen los arboles de jerarquía mediante la inclusión de cada fenómeno en un criterio de evaluación. Los criterios de evaluación seleccionados son los contextos en los que están inmersos los datos espaciales, la composición y funcionamiento de las geocomunidades que operan con los datos y el rol de la sociedad.

Figura 2. Árbol de jerarquía estructural de la producción y consumo en la Geoesfera

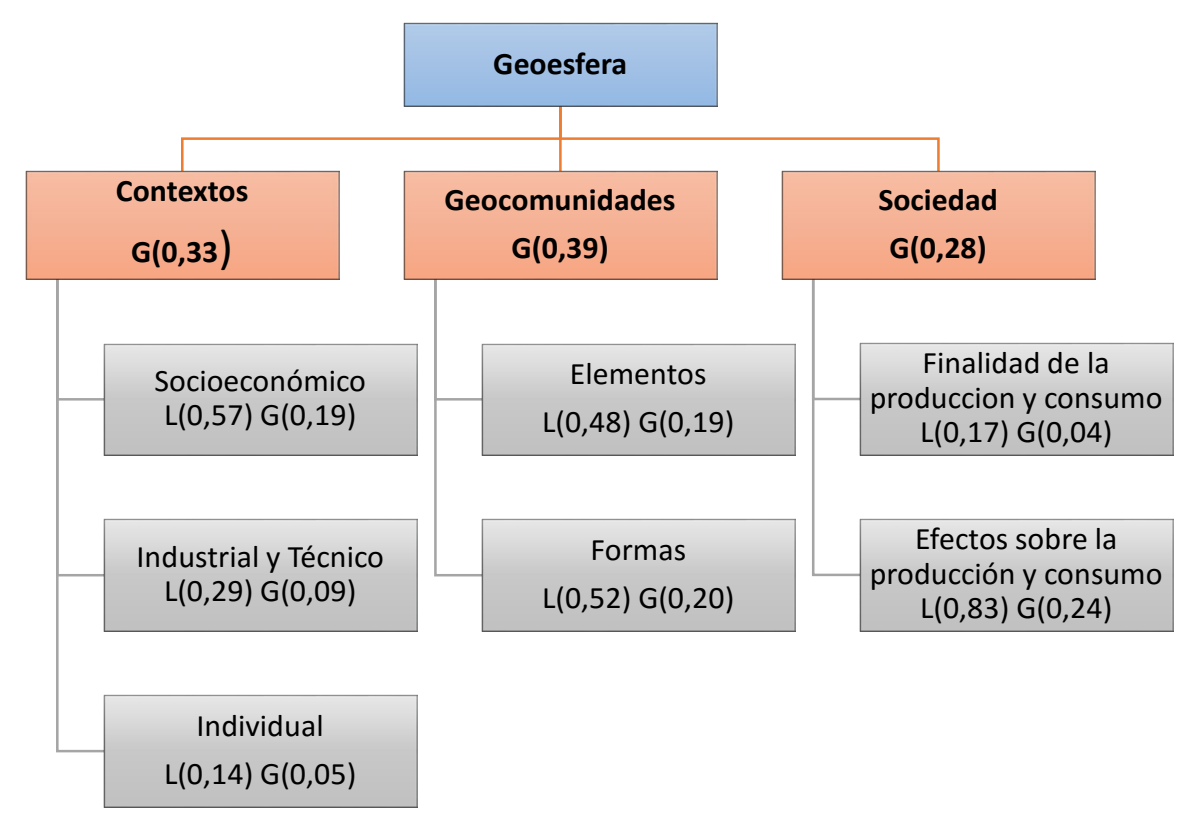

Nota: Los macrocriterios están señalados en cuadros de color anaranjado. Los criterios en cuadros grisáceos. $L$ es el peso local y $G$ es el peso global del criterio.

FUENTE: Elaboración del autor 
Los criterios se han agrupado en macrocriterios de evaluación estructural (FIGURA 2), que describen las fuerzas conductoras que operan en el uso presente de los datos espaciales. Esta organización de los macrocriterios no es única; la geoesfera admite también una aproximación funcional que distinga producción y consumo como hemos visto en la introducción (FIGURA 3).

Las alternativas a evaluar son los paradigmas de producción con el fin de determinar cómo estos modos de producción y consumo son capaces de satisfacer los fenómenos actuales.

Figura 3. Arbol de jerarquía funcional de la producción y consumo en la Geoesfera

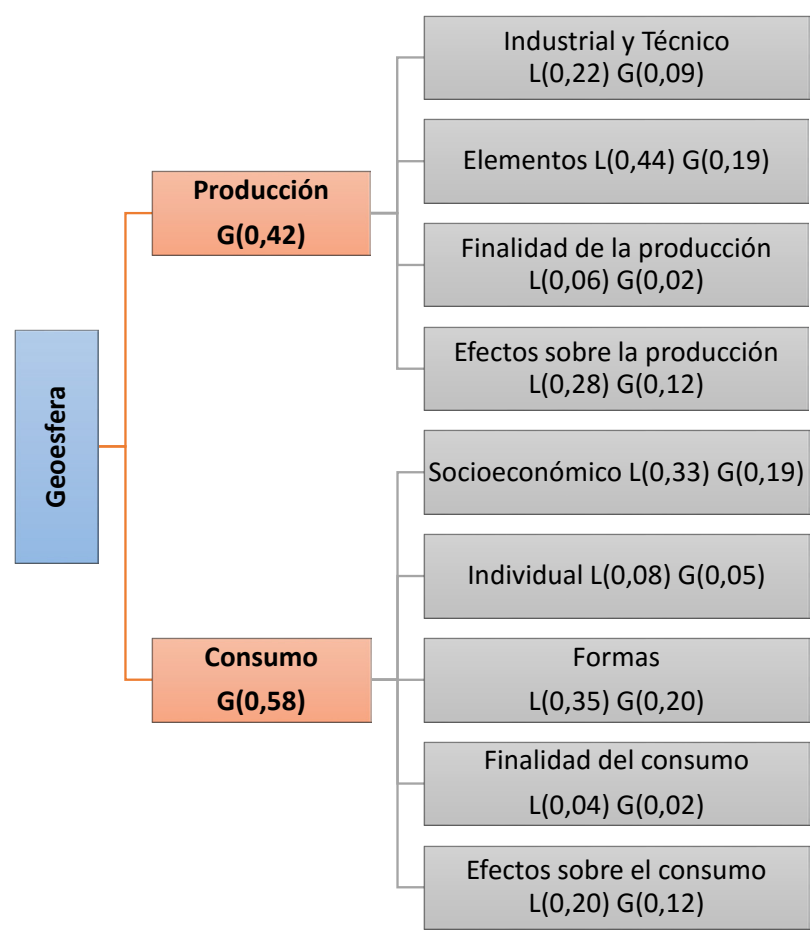

Nota: Los macrocriterios están señalados en cuadros de color anaranjado. Los criterios en cuadros grisáceos. $L$ es el peso local y $G$ es el peso global del criterio.

FUENTE: Elaboración del autor

Para obtener la puntuación de cada alternativa en cada criterio, se asigna a cada fenómeno el paradigma o el conjunto de paradigmas que mejor resuelven el reto planteado, de tal forma que la suma de paradigmas nos ofrezca la puntuación de cada alternativa, la cual se normaliza para poder comparar criterios 
con distinto número de fenómenos. El criterio de normalización seleccionado es el del porcentaje del total para mantener el orden pre y post normalización y la visión de la composición de paradigmas en cada criterio.

En la matriz de evaluación se recoge el número de paradigmas normalizado que integra cada criterio (CUADRO 4). En ella se aprecia la contribución de cada paradigma de producción para satisfacer los criterios que hemos identificado y que describiremos en profundidad en los siguientes apartados. Los principios del sistema Agile son los que satisfacen en mayor medida los fenómenos sucedidos en la geoesfera. A pesar de esta dominancia, se observa una situación ecléctica en la que todos los paradigmas vigentes de producción participan en mayor o menor grado para satisfacer los fenómenos actuales. Es decir se observa la existencia de una vía ecléctica, en la que para satisfacer el conjunto de fenómenos que se han producido en la geoesfera, es preciso recurrir a todos los paradigmas productivos.

Cuadro 4. La matriz de evaluación

\begin{tabular}{|c|c|c|c|c|c|c|c|c|c|c|}
\hline 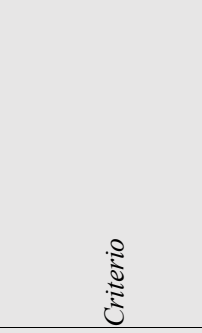 & 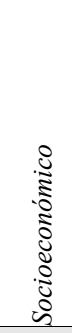 & 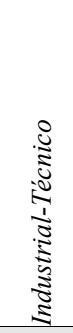 & 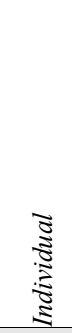 & 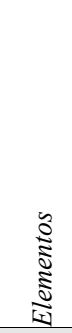 & 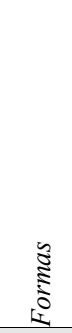 & 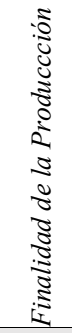 & 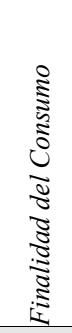 & 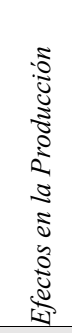 & 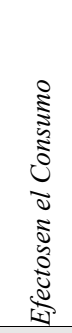 & $\frac{7}{7}$ \\
\hline Alternativa/Peso & 0,19 & 0,09 & 0,05 & 0,19 & 0,2 & 0,02 & 0,02 & 0,12 & 0,12 & 1,00 \\
\hline$A$ & 0,25 & 0,25 & 0,25 & 0,13 & 0,24 & 0,50 & 0,00 & 0,30 & 0,30 & 0,24 \\
\hline C & 0,13 & 0,25 & 0,25 & 0,13 & 0,24 & 0,00 & 0,50 & 0,10 & 0,20 & 0,18 \\
\hline$L$ & 0,19 & 0,13 & 0,25 & 0,13 & 0,06 & 0,00 & 0,00 & 0,10 & 0,00 & 0,11 \\
\hline$R C$ & 0,13 & 0,13 & 0,00 & 0,06 & 0,18 & 0,00 & 0,00 & 0,20 & 0,20 & 0,13 \\
\hline$S$ & 0,19 & 0,00 & 0,00 & 0,19 & 0,12 & 0,50 & 0,00 & 0,00 & 0,10 & 0,12 \\
\hline$W$ & 0,06 & 0,13 & 0,25 & 0,13 & 0,12 & 0,00 & 0,50 & 0,10 & 0,20 & 0,13 \\
\hline$R E$ & 0,06 & 0,13 & 0,00 & 0,13 & 0,00 & 0,00 & 0,00 & 0,20 & 0,00 & 0,07 \\
\hline$G$ & 0,00 & 0,00 & 0,00 & 0,13 & 0,06 & 0,00 & 0,00 & 0,00 & 0,00 & 0,04 \\
\hline
\end{tabular}

Nota: Agile (A), Cloud (C ), Lean (L), Reconfigurable (RC), Sustainable (S), Web 2.0. (W), Resilience Resistant (RE), Green (G),

FUENTE: Elaboración propia 
El cociente de consistencia para todas las matrices es superior al $95 \%$ y la sensibilidad ante la aparición o desaparición de un fenómeno es del 1,18\% en los pesos y puntuaciones finales (FIGURA 4).

Figura 4. Análisis de sensibilidad. Variación en tanto por uno de los pesos en los criterios y en la puntuación final de las alternativas por la variación del número de fenómenos

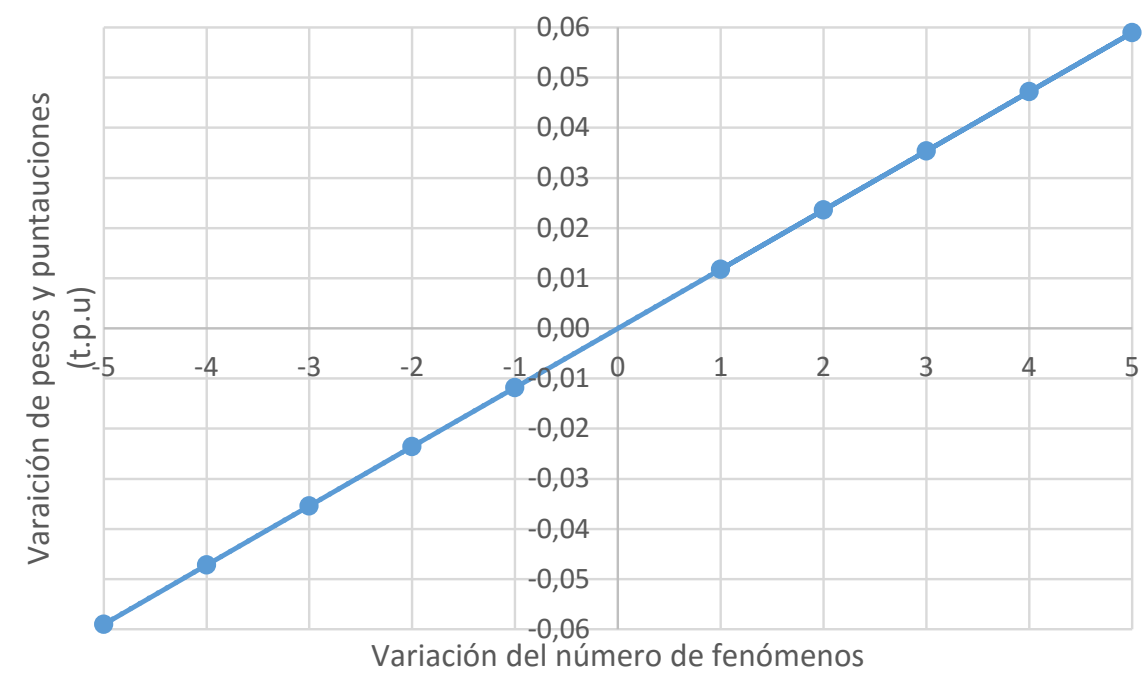

FUENTE: Elaboración del autor

\section{LOS CONTEXTOS DEL DATO ESPACIAL}

\section{IV.1. El contexto socioeconómico}

Los datos espaciales no son ajenos al marco social existente, sino que son parte de la reinante sociedad de la información y participan de la liquidez que describe el momento presente, regulado por una economía de la atención que ha sido descrita como la cultura del aperitivo. Este trinomio se adapta bien al paradigma «Agile» de producción y al consumo de datos espaciales (CUADRO 4). Este esquema está regulado por un consumo condicionado por la economía de la atracción, que busca el valor de uso o de cambio mediante la transformación del dato en información, y ésta en conocimiento geográfico.

El contexto socioeconómico ha provocado que el consumidor actual tenga unas expectativas concretas sobre los datos espaciales: requiere de datos espaciales veraces y exactos, en múltiples formatos, rápidamente accesibles, fáciles de usar, integrables en dispositivos y plataformas, actualizados y comprensibles. Este ideario del consumidor moderno se puede resumir en que 
plantea al productor la necesidad de obtener datos adecuados al fin que precisa y que estén servidos en el instante y forma solicitados. En el cuadro 5 se presenta cómo ha reaccionado la producción frente a estas demandas.

Cuadro 5. El contexto socioeconómico: la reacción de la producción frente a la demanda de datos espaciales

Proceso social y

tipo de consumo

Reacción de la producción

\section{Modernidad líquida}

(BAUMAN, 2000)

El consumo urgente Induce la velocidad en la generación y actualización del dato.

El consumo de multi- Consolida la interoperabilidad, la optimización de serfuentes vicios y el desarrollo de apps.

El consumo del cambio Impulsa la cartografía 4D y las animaciones cartográficas.

\section{La cultura del aperitivo}

(NEWMAN, 2010)

El consumo rápido Fomenta la destilación del dato en información y conocimiento mediante el desarrollo de modelos espaciales.

El consumo de experien- Busca un contexto narrativo que acompañe al dato escias pacial.

El consumo condensado Requiere de una facilidad en la digestión de la información espacial que se logra mediante un renacimiento del diseño, que pasa a estar centrado en el usuario (TSOU, 2011), como reacción al diseño clonado de mapas (DEL RÍO, 2009).

\section{La sociedad de la información}

(CASTELls, 2004)

El consumo global Provoca una universalidad de la simbología (KORPI y AHONEN-RAINIO, 2010).

El consumo multiplata- Adapta el diseño a las características de cada disposiforma tivo (screen-mapping, web mapping, mobile mapping) y a la cartografía ubicua (GARTNER et al., 2007).

La reutilización del dato Desarrolla las políticas y las licencias de datos.

El omnivorismo cultural Consume todo tipo de datos espaciales. (PETERSON, 1992)

\section{La economía de la atención}

(DAVENPORT y BECK, 2002):

La búsqueda del con- Crea un duelo de bases de datos, formatos de ficheros sumo y geowebs. 
$\begin{array}{ll}\text { La intermediación geoes- } & \text { Hace que nazcan intermediarios geoespaciales por la } \\ \text { pacial } & \text { geo-infointoxicación y la fragmentación de datos, entre } \\ & \text { los que destaca: los geoportales, los metadatos, la blo- } \\ & \text { gosfera, las redes sociales y los buscadores generalistas } \\ & \text { y especializados en Internet. Se debate sobre el segui- } \\ & \text { miento de usuarios y la privacidad. }\end{array}$

La economía de la atracción

(ROBERTS, 2005)

La personalización del Desarrolla múltiples servicios sobre los datos en funconsumo

ción de la capacitación geoespacial y la finalidad del consumo.

La seguridad del con- Desencadena un debate sobre la seguridad jurídica, las sumo garantías técnicas y la responsabilidad de los productores.

La confianza en el con- Amplía el modelo de calidad de los datos más tradiciosumo nal hasta añadirse otros indicadores como la confianza o la reputación.

La satisfacción del con- Es el nuevo indicador clave de desempeño. sumidor

La desclasificación entre Genera la imagen de marca del productor.

los datos neogeográficos

y los profesionales

FUENTE: Elaboración del autor

\section{IV.2. El contexto industrial y técnico}

El dato espacial, además del carácter continuo, presenta hoy en día unas características propias (CUADRO 6) que lo diferencian de cualquier otro producto de naturaleza cartográfica que hayamos elaborado con anterioridad. El nacimiento de estas nuevas características se debe tanto a las posibilidades productivas que brinda la tecnología como a la manera en que han sido articuladas por la industria. Los paradigmas Agile y Cloud son los que mejor se adaptan a estos fenómenos (CUADRO 4)

Cuadro 6. El contexto industrial y técnico. Características del dato espacial en Internet

La digitalización La informática y el desarrollo tecnológico comenzaron un del dato proceso de abaratamiento de los costes de obtención y procesamiento de los datos espaciales, así como de la confección de mapas.

La visibilidad del El aspecto más destacado proporcionado por la Red es el nadato: Internet cimiento de plataformas para hacerlos visibles, dos de las más utilizadas son los geo-portales y los sistemas de metadatos. 


\begin{abstract}
El dato se puede crear y compartir: la web 2.0 y las redes sociales.
\end{abstract}
Las herramientas amparadas en la etiqueta de la web 2.0 ofre- cen un nuevo sistema que es complementado con las tecnolo- gías colaborativas. Este binomio brinda la posibilidad de edi- tar y compartir los datos. Estas tecnologías socializan los me- dios de producción y ayudan a normalizar su entrada masiva en la sociedad.

El dato se puede Las nuevas tecnologísa y la accesibilidad a Internet proportransformar y re- cionan al usuario acceso a bases de datos espaciales, mapas utilizar base o software gratuito. Todo ello facilita la producción, distribución, transformación, reutilización y análisis del dato mediante MASups, APIS, Widgets, APPS tanto en software propietario como libre. Esto favorece la equidad en el acceso a usuarios con distintas características sociodemográficas y de capacitación geoespacial.

La globalización La necesidad de integrar datos ha creado, a su vez, la necesidel dato dad de asegurar la interoperabilidad de la información geoespacial; para ello se crean servicios que permiten reunir y utilizar la información elaborada por distintos productores, que se pone a disposición en múltiples dispositivos y plataformas.

El fenómeno in- Los artefactos, los productos, las personas, las organizaciones, clusivo del dato y los seres vivos, generan geodatos. La geolocalización es un espacial, «geo- atributo asociado al dato que se vuelve imprescindible para todo» analizar la realidad. Estamos ante un paisaje de datos como producto de la actividad humana, que GOODCHILD (2009b) resume en la frase «conocer donde está todo en cada momento». Algunos ejemplos recientes son la denominada sensorización del mundo, el Internet de las cosas, el big data, la geocodificación, los mapas de etiquetas que permiten la navegación por folcksonomías o resumir la búsqueda de tendencias, la realidad virtual, datos en tiempo real para la gestión de las ciudades inteligentes, la cartografía de la ciencia, la integración de datos sociales locales y móviles (SoLoMo), los sistemas basados en localización (LBS), la geoposición en interiores, o el geodiseño entre otros.

La usabilidad y Se produce un avance en la usabilidad del dato y en la amabiportabilidad del lidad de la aplicación para el usuario final mediante patrones dato de soluciones accesibles y portables.

El dato es espa- El dato tiene un ciclo de vida. La longevidad del dato viene ciotemporal determinada por el ritmo de cambios que se producen en la realidad descrita por ellos.

FUENTE: Elaboración propia

\section{IV.3. El contexto individual}

En el nivel individual (FIGURA 5) intervienen dos factores: la motivación y los capitales. 
La motivación responde a las siguientes cuestiones: ¿para qué usamos los datos espaciales?, ¿cuál es la finalidad de su consumo? En este punto las motivaciones del consumo están muy influidas por el valor de uso y el valor de cambio que conferimos a los datos espaciales. La motivación elabora una demanda de datos espaciales que está mediada por el contexto socioeconómico. La demanda toma forma en la intersección de la finalidad del consumo con el contexto socioeconómico, es decir, es el resultado de un balance entre el determinismo del contexto y el constructivismo de la motivación.

Los capitales son el conjunto de recursos de los que dispone el usuario para satisfacer la demanda mediante el uso de las posibilidades tecnológicas que le brinda el contexto técnico-industrial. El uso factible de la producción es la intersección de los capitales del individuo con la oferta facilitada por el contexto técnico industrial, es decir, es el resultado de un balance entre el determinismo del contexto y el constructivismo de los capitales.

Figura 5. La capacitación geoespacial

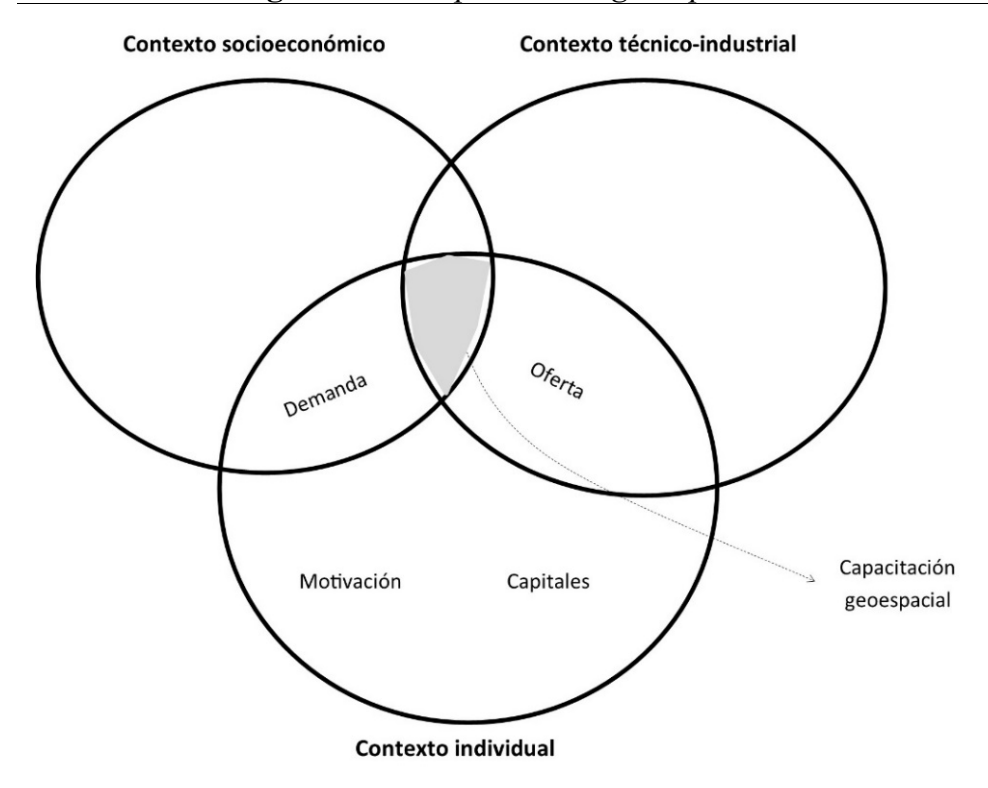

FUENTE: Elaboración del autor

La capacitación geoespacial es una capacidad o habilidad, consecuencia del proceso de adquisición del conjunto de competencias necesarias para la utilización motivada de los datos espaciales en un contexto socioeconómico y técnico-industrial concreto. Por lo tanto, no se refiere exclusivamente a los capitales, incluye también a las motivaciones. Ambas son puestas en juego por el 
individuo en unos contextos concretos que determinan las demandas de uso y las posibilidades tecnológicas que permite la producción. El consumo está mediado por la capacidad geoespacial que posee el individuo, y que nace en la intersección de los tres contextos.

Entre los capitales podemos distinguir varios ejes: formativos, institucionales, sociales, económicos y culturales (FIGURA 6). La agregación de todos ellos junto con sus motivaciones nos permite conocer cuál es la habilitación de un sujeto en la producción y consumo de la información geoespacial.

Figura 6. Los capitales de la capacitación geoespacial

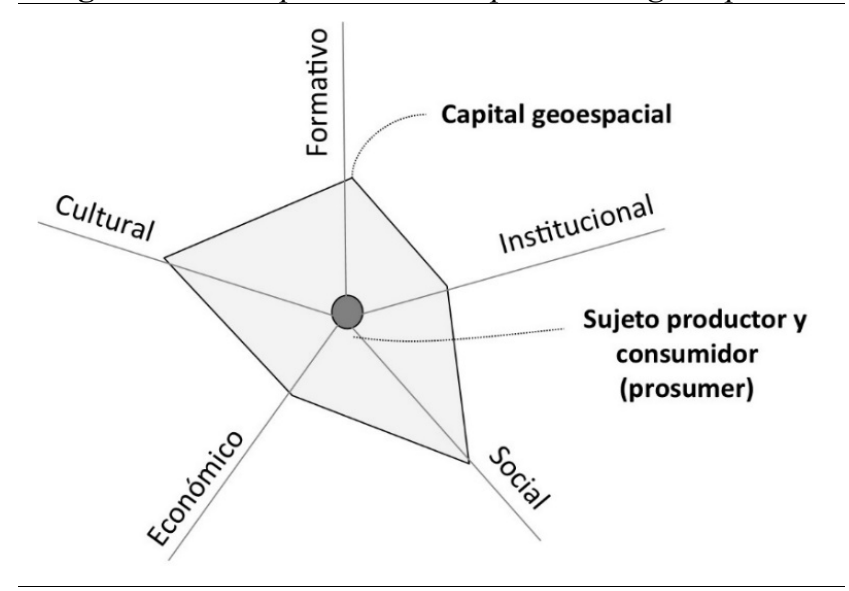

FUENTE: Elaboración del autor

Los paradigmas Agile, Cloud, Lean y Web 2.0 son los que satisfacen el mayor número de fenómenos observados en el contexto individual (CUADRO 4).

\section{LAS GEOCOMUNIDADES: ELEMENTOS}

Los elementos principales que intervienen en el proceso de producción y consumo de datos espaciales se presentan en la FIGURA 7.

A continuación vamos a inventariar los principales fenómenos observados en cada tipo de elemento. El paradigma que satisface mayor número de fenómenos es el Sustainable (CUADRO 4).

\section{V.1. El Objeto de consumo: el dato espacial}

\section{V.1.a. El dato espacial está inmerso en geocomunidades}

El objeto de consumo es el dato espacial que se encapsula en la web geoespacial. La cual opera principalmente en torno a geoportales en Internet. Los 
geoportales son el agente de intermediación que distribuye el dato en forma de servicios, ofreciendo una funcionalidad sobre la información. Los geoportales son controlados por geocomunidades. Las geocomunidades también son un agente de mediación, que realiza en mayor o menor medida una trasformación del dato en información o en conocimiento.

Figura 7. Elementos que intervienen en el proceso de producción y consumo

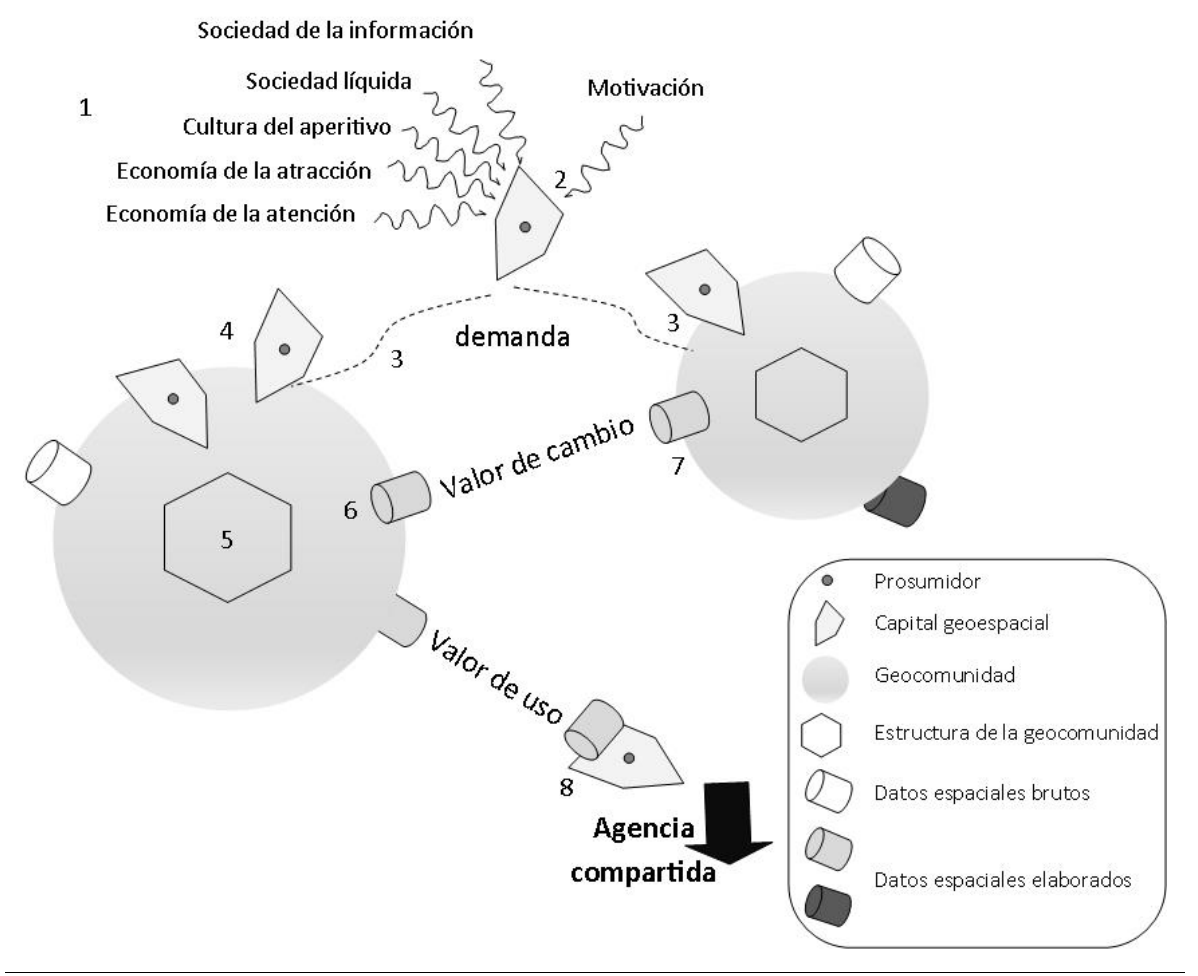

Nota El sujeto de consumo (2), motivado por sus intereses y condicionado por los contextos socioeconómicos, técnicos (1), realiza una demanda de datos espaciales (3). Para satisfacer la demanda los prosumidores eligen y acuden a una geocomunidad, con la que se vinculan en distinta intensidad y grado (4). Las geocomunidades son sistemas sociotécnicos que pueden ser explicadas como sistemas actor-red (5). Las geocomunidades median la producción y el consumo mediante la trasformación del dato en bruto en información o conocimiento (6). A partir de ese momento se abren dos posibilidades: La primera es la utilización del dato elaborado por una geocomunidad como dato en bruto de otra, en un proceso continuo, que materializa el valor de cambio de la información (7). La segunda es la realización de una agencia compartida (8) que materializa el valor de uso del dato espacial. FUENTE: Elaboración del autor 


\section{V.1.b. El dato es interoperable}

Las iniciativas creadas por diversos actores institucionales, gubernamentales, empresariales, universitarios e investigadores, han proporcionado la disponibilidad de múltiples formatos de ficheros y lenguajes de programación. Esta diversidad tecnológica de modos de desarrollo, pese a estar en un proceso permanente de competencia y clausura, ha estado vinculada desde muy temprano al mantra de la interoperabilidad. La interoperabilidad ha sido básica en el desarrollo de la web geoespacial, ha permitido la creación y utilización de estándares espaciales y la integración de datos de distinto origen y formatos de manera rápida, reduciendo los costes de transacción. También ha favorecido la relación entre los sistemas de información.

\section{V.2. El sujeto de consumo: el prosumidor}

\section{V.2.a. El consumidor Beta}

El número y tipo de consumidores ha aumentado y se ha diversificado, pero el cambio más llamativo se ha producido por la desaparición de la concepción clásica de consumidor como receptor que utiliza un producto cartográfico acabado. La cartografia continua hace que se consuman datos que no están completamente actualizados y validados. Se introduce así un consumo de datos espaciales en beta, en permanente y constante perfeccionamiento y actualización, que se caracterizan por sucesivas versiones. En este escenario es el consumo el que demanda e impulsa el ritmo de la producción.

El consumo en beta favorece una comunicación entre productores y consumidores, que es visible al resto del entorno. La transparencia se logra debido a que el consumidor requiere de mayor información sobre el valor de uso e intercambio de los datos para orientar sus decisiones. Esta asesoría es una intermediación que actúa como un «comisario de datos espaciales» y que es asumida por el productor y el consumidor. Desde el lado de la producción la intermediación se realiza mediante el enriquecimiento de los metadatos, a los que se añade un mayor número de ítems y descripciones sobre la calidad y el linaje. Desde la perspectiva del consumo, comienzan a utilizarse indicadores de reputación, confianza y sistemas de recomendación que son consultados desde redes sociales y blogs.

\section{V.2.b. El consumidor interactivo, ubicuo y permanente}

A los consumidores se les ofrecen herramientas accesibles, gratuitas o de bajo coste, con mayor número de opciones para operar con los datos. De la simple consulta se ha pasado a la posibilidad de interactuar con ellos. Ahora pueden realizar operaciones técnicas con un relativo bajo coste de aprendizaje, 
editar, transformar o analizar. También pueden realizar operaciones sociales, visibles para todos, como compartir, comentar, valorar o recomendar la información. El consumidor es interactivo y proactivo; accede a estas operaciones integrando múltiples fuentes de datos que tienen distintos proveedores y lo hace desde variados canales y plataformas.

\section{V.2.c. El prosumidor}

Un mismo sujeto puede desempeñar roles de consumidor y productor. Se desdibuja el rol del consumidor puro ya que los consumidores son a su vez productores de datos. Se habla de prosumidores. Y se identifican cuáles son las diferencias principales de los prosumidores con respecto a los productores clásicos. BRUNS (2008) concreta las diferencias en cinco ámbitos: comunidad, roles fluidos, artefactos en beta, y propiedad común.

Se han planteado múltiples clasificaciones de los usuarios desde distintos tipos de geocomunidades. Cada una de las clasificaciones se centra en un criterio concreto: los roles de producción/uso y experto/aficionado de BUDHATHOKI et al., (2008), la responsabilidad legal y competencia de COLEMAN et al., (2009), la motivación de STREILEIN et al., (2010), el rol en la producción de mapas neogeográficos de MOSEME y VAN ELZAKKER (2012), la responsabilidad en la especificación de los datos y el tipo de datos que capturan de COOPER et al., (2011), el número, frecuencia y longevidad de la edición de mapas de BUDHATHOKI Y HAYTHORNTHWAITE (2013), el poder (entendido como libertad o limitaciones normativas) y la capacidad (definida como número de interacciones que realiza) de GRIRA et al., (2010), o la humanidad, frecuencia, veracidad, y reputación (ANTHONY et al., 2007; ORTEGA y BARAHONA, 2007; PRIEDHORSKY et al., 2007).

La mayor parte de estas calificaciones se adentran a distinguir y etiquetar los distintos tipos de neogeógrafos o voluntarios de información geográfica, pero las evidencias empíricas halladas por BUDHATHOKI y HAYTHORNTHWAITE (2013) ponen en tela de juicio la generalización de esta dicotomía. Un dato llamativo es que en $O S M$ casi un $70 \%$ de los usuarios que editan tiene formación universitaria y un $50 \%$ tiene experiencia profesional con GIS.

\section{V.2.d. La satisfacción del consumidor}

La calidad no es fácilmente certificable pues depende de los contextos culturales (BECK, 2005), del destinatario y del uso al que se destinen los datos. Para solventarlo se proponen nuevos modelos de evaluación, que rehúyen de los términos técnicos tradicionales basados exclusivamente en la calidad de la 
información. Las nuevas valoraciones se orientan hacia un concepto más global: el de satisfacción del consumidor (MOSEME y VAN ELZAKKER, 2012).

\section{V.3. La economía de la vía ecléctica}

\section{V.3.a. El dato espacial es objeto de inversión}

La nueva economía del dato espacial se caracteriza porque el objeto de consumo es una mercancía de información, cuya generación y mantenimiento implica una inversión económica. En este marco conceptual se puede analizar la inversión mediante las métricas clásicas de evaluación económica de proyectos que consideran los costes y los beneficios.

En cuanto a los costes, las fases de recopilación y de mantenimiento tienen unos costes fijos altos. En cambio, las fases de reproducción y difusión avanzan hacia un coste marginal nulo. Por lo que respecta a los beneficios, estos van aumentando según se van refinando los datos y se transforman en información y conocimiento. El dato tiene un ciclo de vida y progresivamente disminuye su valor en el tiempo según se desactualiza hasta que vuelve a repuntar su valor como dato histórico o perteneciente a una serie temporal.

\section{V.3.b. La distribución de los costes productivos}

La accesibilidad y los requerimientos normativos han hecho que el dato espacial sea producido por quien está más próximo al dato. El principio de cercanía productiva no sólo es aplicable desde la competencia jurídica, también lo es geográficamente, especialmente en las labores realizadas por los neogeógrafos.

La aplicación del principio de cercanía productiva implica una fragmentación y atomización de la producción de los datos y, por consiguiente, una disminución de los costes por actor. El reparto en las tareas de producción es la base de la racionalización en la asignación de los recursos. Sin embargo, las consecuencias no son sólo económicas ya que hay otros efectos como la reducción del Gap-GIS (DEL RIO, 2012a) que se produce al intervenir un mayor número de actores, y la creación de múltiples geocomunidades que gestionan la producción y el consumo. La desventaja de la división de la producción es que puede provocar a corto plazo un desabastecimiento en el mercado. La ausencia de datos deriva en muchas ocasiones de la siempre difícil tarea de implantar y mantener operativa una geocomunidad.

\section{V.3.c. El valor sustituye al precio}

La economía del dato espacial plantea una ruptura con la economía tradicional de la información geográfica, debido a que el valor de uso e intercambio 
se convierten en protagonistas, en detrimento del valor de mercado. Las características del dato espacial en Internet, que exponíamos en el CUADRO 6, han propiciado la reutilización del dato espacial y son la causa de los menores costes marginales.

Las consecuencias económicas del cambio de modelo de producción y consumo son profundas y plantean una crisis a los modelos de negocio tradicionales basados en la idea de vender la información o facturar por los servicios geoespaciales. Los modelos más afectados son los que necesitan de una gran cantidad de datos, lo cual es costoso y dificulta la aparición de una masa crítica de consumidores que la haga rentable. Además, actualmente en Internet, los consumidores han desarrollado hábitos de selección de contenidos gratuitos o que requieren un pago mínimo.

\section{V.3.d. La amortización del dato espacial}

La pugna por disponer de recursos económicos o rentabilizar inversiones, ya no se puede plantear exclusivamente en base a precios de mercado, sino de utilidades. No pedimos a la economía que trate de fijar el precio del producto; su nueva misión es cuantificar el éxito de implantar y mantener una geocomunidad que gestione datos espaciales, o valorar el fracaso que supone carecer de ella (KURWAKUMIRE, 2014). Esta misión es crucial porque la eficacia y eficiencia económica del dato espacial influyen en el grado en que los datos espaciales se pueden adquirir, utilizar y distribuir (DESSERS et al., 2012).

La pregunta clave que nos planteamos es ¿cuánto cuesta tomar a tiempo buenas o malas decisiones? Para responder a esta cuestión comienzan a desarrollarse metodologías de evaluación (KURWAKUMIRE, 2014) que se centran en qué evaluar y cómo medir los beneficios, especialmente los denominados intangibles.

\section{V.3.e. El encapsulamiento del beneficio de los datos espaciales}

Habitualmente, en las evaluaciones económicas, el enfoque de proyecto de inversión es el más utilizado. Sin embargo, este marco tiene dificultades prácticas ya que el valor de uso y el valor de cambio están encapsulados en otros procesos productivos, muchas veces concatenados, lo que hace que el beneficio sea difícil de cuantificar, especialmente desde el momento en que hemos comenzado a producir ingentes cantidades de nuevos datos, que incorporan el atributo espacial proporcionado por la huella de todo tipo de actividades y dispositivos. 
Para solventar la evaluación DESSERS et al. (2012) plantean un modelo que tiene en cuenta todo el proceso que se gestiona con la ayuda de los datos espaciales. La hipótesis del modelo es que no se puede separar el dato del proceso en el que está inmerso. El encapsulamiento del dato permite que el rendimiento de una SDI se mida por la capacidad que tiene de trabajar con un proceso en el que existe una componente espacial. JANSSEN (2011) plantea que el valor también es función de la importancia que el fabricante, el legislador o la política conceden a la creación, a la disponibilidad y al acceso a estos datos para la democracia y la acción de gobierno.

\section{V.3.f. El rendimiento de las geocomunidades de datos espaciales}

Las geocomunidades son más que un proyecto de inversión, son una organización que tiene un funcionamiento continuado y en permanente evolución en el tiempo. Estas características obligan a concebir enfoques que se adapten más al rendimiento global de la geocomunidad. En esta línea de trabajo GIFF y CROMPVOETS (2008) proponen una metodología de evaluación estructurada desde múltiples puntos de vista con indicadores de desempeño como herramienta para medir e informar de la actuación de una SDI.

\section{LAS GEOCOMUNIDADES: LAS FORMAS DE PRODUCCIÓN Y CONSUMO}

La forma de producción y consumo hace referencia a dónde y cómo se realizan estas actividades.

El consumo de la información espacial es tecnológica y económicamente accesible por gran parte de los ciudadanos y de las organizaciones desde variados dispositivos, lo que ha aumentado y diversificado los patrones que definen cómo se realiza el consumo.

La oferta de contenidos ofrecida al consumidor es centralizada por geocomunidades. En ellas, cobra un gran protagonismo el valor de cambio de los datos en información y en conocimiento geoespacial. Hoy en día el consumo de datos sirve a su vez de materia prima a nuevos procesos de producción. La concatenación de los ciclos productivos sucesivos sirve para diferenciar y caracterizar los lugares donde se realiza la producción y el consumo.

Los paradigmas que satisfacen mayor número de fenómenos son el Agile y el Cloud (CUADRO 4). 


\section{VI.1. Las geo-comunidades}

Las geocomunidades realizan una mediación de los datos existentes, dentro de las actividades sociales (HAKLAY et al., 2008). No sólo son un marco tecnológico en torno al dato espacial, son también una infraestructura sociotécnica (DODGE et al., 2009) que puede ser analizada en base a la teoría del actorred (DE MAN, 2007). El individuo participa como actor humano en más de una geocomunidad con distintos roles e intensidad en sus vínculos.

Las geocomunidades son el ágora en el que se efectúa la negociación del proceso de producción y consumo de los datos espaciales. Es una red de actores que realizan una agencia compartida, cuyo éxito se puede resumir en términos de su estabilidad o supervivencia en el tiempo.

La aparición de estas redes se debe en gran parte al omnivorismo espacial de nuestra realidad, que ha provocado la necesidad de agentes y sistemas intermediarios que faciliten el acceso a la información, reduciendo los riesgos y maximizando la confianza en el consumo.

En la FIGURA 8 se recogen los principales elementos que permiten definir una geocomunidad, aunque esta perspectiva estructural no sea el único enfoque posible. DE MAN (2007) propone una caracterización en base a los desafíos, conceptos clave, perspectivas comunes y dilemas que presentan las $S D I$ frente a otros tipos de sistemas de información.

Los antecedentes históricos (WENGER, 1998; NOUCHER y GOLAY, 2010) y los factores que motivan el nacimiento de las geocomunidades son las áreas más investigadas (WENGER, 1998; GEORGIADOU et al., 2007; COLEMAN et al., 2009; CREIGHTON, 2005; GRAHAM, 2011; ElWOOD, 2011), aunque no las únicas. Otros autores se han centrado en analizar la organización (TURNER y GORMAN, 2013), la actividad de los miembros (NEIS y ZIPF, 2012), la visión (GORMAN, 2009), el valor (NOUCHER y GOLAY, 2010), e incluso se ha empezado a trabajar en predecir su posible evolución en el tiempo (DE MAN, 2007).

Ya se han realizado las primeras tentativas de clasificación de tipos de geocomunidades (COLEMAN et al., 2009). Algunas clasificaciones se centran en aspectos organizativos como la diferencia entre organizaciones y comunidades que plantean TURNER y GORMAN (2013), mientras que otros se centran en aspectos estructurales en función del tipo de datos y sus especificaciones (COOPER et al., 2011).

También se han realizado observaciones sobre las geocomunidades surgidas en ámbitos temáticos concretos. Tal es el caso de los trabajos centrados en los episodios de crisis y emergencias (LIU y PALEN, 2010), en Open Street Map 
(NEIS y ZIPF, 2012), en las comunidades web 2.0 (BRUNS, 2008), en las geocomunidades de carácter local mantenidas por organizaciones no gubernamentales (ELWOOD, 2011), en las relacionadas con las SDI, o en los GIS de participación ciudadana, PPGIS (ATZMANSTORFER y BLASCHKE, 2013).

Figura 8. Estructura de las geocomunidades

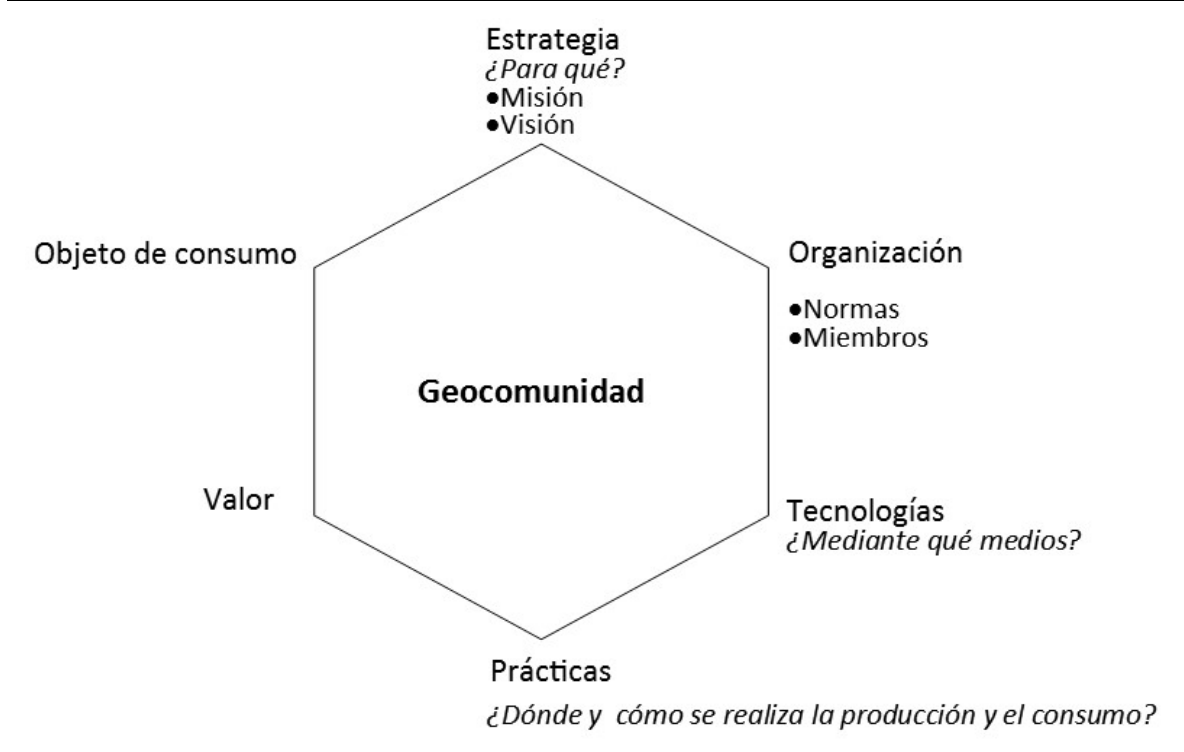

FUENTE: Elaboración del autor

\section{VI.2. La geoweb}

La geoweb es coral (GOODCHILD, 2007) y está formada por muchas geocomunidades. En este ecosistema las geocomunidades desarrollan relaciones inter e intraorganizativas mediante políticas, acuerdos y licencias. Establecer estas relaciones tiene una gran ventaja: permite destinar los recursos hacia la creación de datos propios y originales mediante la integración y reutilización de la información elaborada por otras geocomunidades. En este sentido se puede afirmar que casi todas las geocomunidades son usuarios de otras.

\section{VI.3. EI mix-productivo}

Existen tres grandes tipos de datos en función de quién sea su productor y la finalidad con la que se coleccionan. Cada uno de ellos presenta unas técnicas de captura, un modelo, un tipo de archivo de la información y un software. 
El primer grupo está formado por datos muy tecnificados y especializados. El uso de este tipo de datos está ligado generalmente a una producción y consumo profesional o científico, y a una reutilización que aporta un gran valor. En este campo se ha producido una hiperespecialización y una reivindicación del valor de los small — data. Un segundo grupo está compuesto por datos generalistas y cotidianos. Están más vinculados con el estilo neogeográfico y de voluntariado de la web 2.0. En este grupo la neogeografía y el voluntariado son las formas habituales de producción y consumo. El tercer grupo proviene de la automatización en la producción, la cual se alcanza gracias y entre otros artefactos, a los sensores, al registro de la huella informática de la actividad cotidiana, al Internet de las cosas, o la informacionalización. En este último grupo la forma que ha tomado la producción y el consumo es el binomio del big data y el geo-smart.

El mix productivo de estos tres tipos de datos está facilitado por los estándares de interoperabilidad, pero la convivencia de los datos no es fácil y está en fase de debate. La disparidad de la calidad, las garantías técnicas y la seguridad jurídica de cada tipo de datos condiciona su integración; especialmente se discute la utilidad de la neogeografía para enriquecer a otros conjuntos de datos. A pesar de estas dificultades se están buscando marcos de integración que superen la segregación inicial.

\section{VI.3.a. La hiper-especialización}

Alrededor del dato espacial se incorporan profesionales de distintas áreas. La informatización y la mayor cantidad de datos disponibles han producido una híper-especialización en las labores de producción y análisis.

\section{VI.3.b. La Neogeografía y el voluntariado de información geográfica}

Se incorporan nuevos actores no profesionales, tanto en las labores de producción como de consumo. Sin embargo, la doctrina presta inicialmente mucha mayor atención a la participación de los no profesionales en las labores de producción. Cualquier persona con conocimientos razonables y acceso a las herramientas de la Web 2.0 puede ser un editor de mapas (CARTWRIGHT, 2012).

Se crea un mercado terminológico alrededor de este fenómeno. Algunos de los términos más utilizados son: voluntariado de información geográfica (VGI) (GOODCHILD, 2007; GOODCHILD, 2009b), neogeografía (TURNER, 2006; BUCKINHAM y DENNIS, 2009; RANA y JOLIVEAU, 2009), nuevos medios espaciales (CRAMPTON, 2009), la geoweb (ELWOOD y LESZCZYNSKI, 2013; HAKLAY et al., 2008), Mapas 2.0 (CRAMPTON, 2009), la wikificación del GIS (SUI, 2008), las GeoWikis (GUPTILL, 2007), y la inteligencia de las multitudes 
(HeIPKE, 2010; DodGE y KiTCHIN, 2013). El fenómeno comienza a ser estudiado desde la ciencia GIS y la cartografía crítica (WARF y SUI, 2010; ELWOOD, 2010; SHEPPARD, 2005).

El papel de la Academia se muestra en la FIGURA 9 que representa la evolución de las publicaciones científicas en artículos indexados en Google Académico. Los datos se han obtenido mediante el software Publish o Perish (HARZING y VAN DER WAL, 2007), que está disponible en http://www.harzing.com/pop.htm. Se ha escogido la base de datos de Google Académico por recoger actas de congresos y revistas no inglesas (MEHO y YANG, 2007). En el análisis se han seleccionado los tres términos más populares para caracterizar la producción científica: neogeografía, voluntariado de información geográfica, y sociedad capacitada geoespacialmente. En el gráfico se observa como el término de voluntariado es el que mayor número de artículos ha generado, a pesar de haber nacido con posteridad al término de neogeografia.

Figura 9. Evolución en el periodo 2003 a octubre de 2014 del número de artículos científicos indexados en Google Académico que hacen referencia a neogeografía, voluntariado de información geográfica y sociedad capacitada geoespacialmente

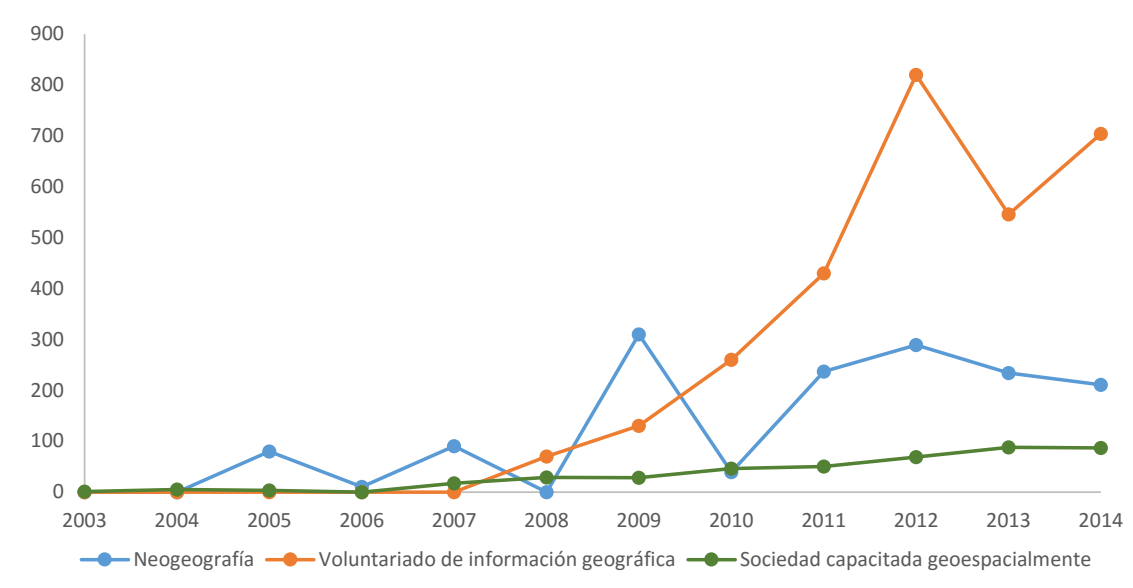

FUENTE: Elaboración del autor

Los conceptos no son equivalentes sino que presentan diferencias entre ellos. La FigURA 10 incluye la cartografía de la ciencia de los tres conceptos. Se ha elaborado a partir de las bases de datos de Google Académico, siguiendo las metodologías descritas por YAO (2014), LEE (2014) y CARDONA 
y SANZ (2015). El resultado se ha representado mediante el software VosViewer (이리 Center for Science and Tech-nology Studies, Leiden University, The Netherlands; libremente disponible en: http://www.vosviewer.com/).

La FIGURA 10 indica que cada uno de estos conceptos tiene distintas áreas de interés. Las áreas de color rojo indican una mayor densidad de palabras clave, las amarillas una densidad intermedia y las verdes una intensidad baja.

El gráfico revela cómo la capacitación geoespacial es un concepto de clausura, claramente conciliador, surgido en el ámbito de las SDI que intentan aprovechar el potencial del voluntariado. La neogeografía y el voluntariado presentan nexos y temas comunes. Sin embargo, la neogeografia se centra más en los términos que hacen referencia al análisis y los casos de uso, mientras que el voluntariado destaca la capacidad de generación de datos y las consecuencias geográficas y sociales del fenómeno. Este análisis concuerda con las diferencias entre ambos términos reseñadas por WILSON y GRAHAM (2013).

Figura 10. Cartografia de la ciencia en el periodo 2003 a octubre de 2014 de artículos científicos indexados en Google Académico referidos a neogeografía, voluntariado de información geográfica y sociedad capacitada geoespacialmente

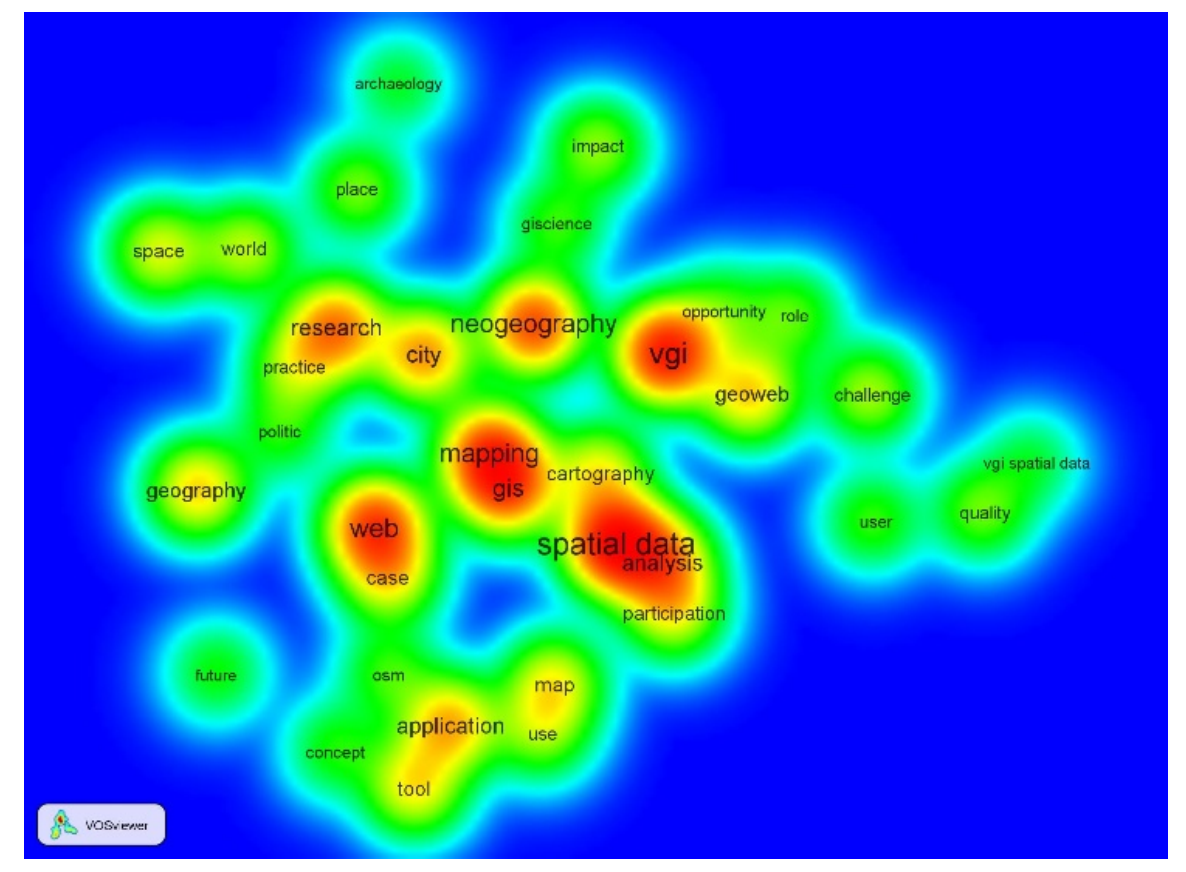

a) Neogeografía 


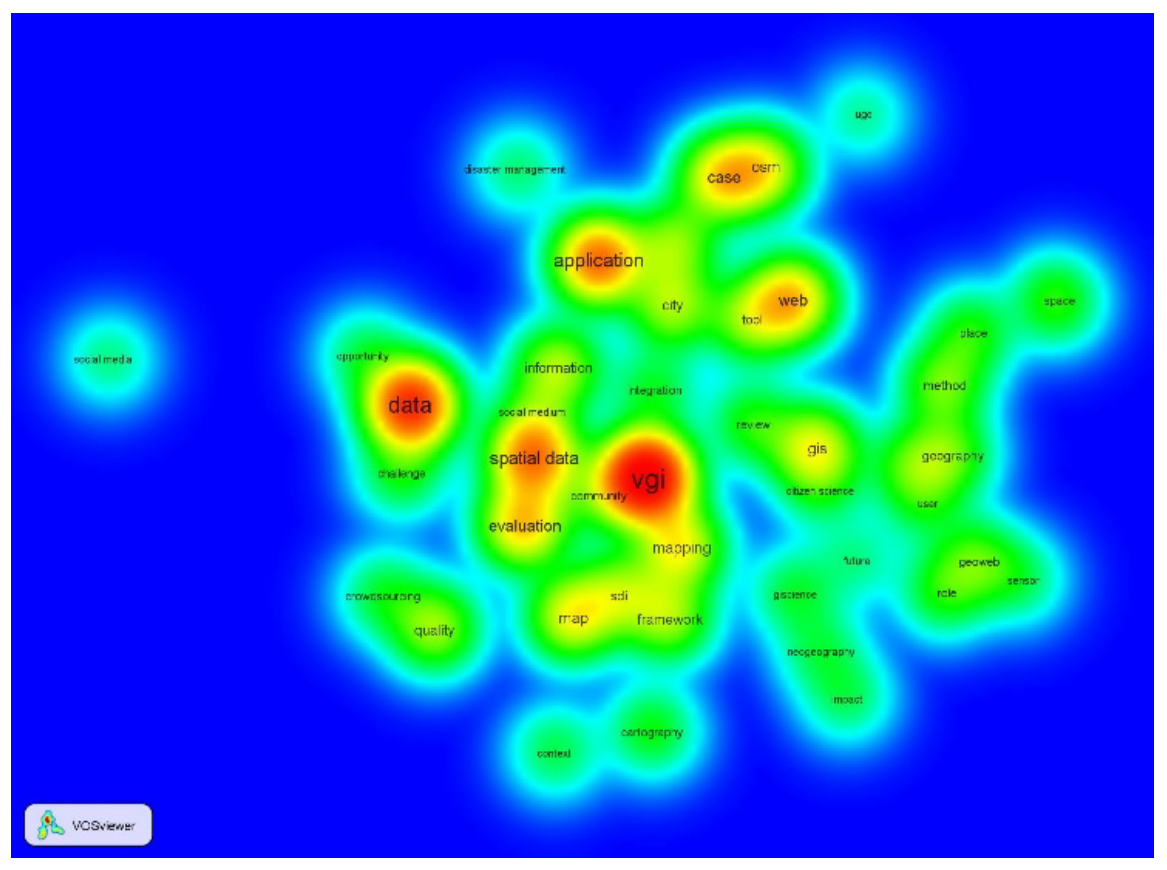

b) Voluntariado de información geográfica

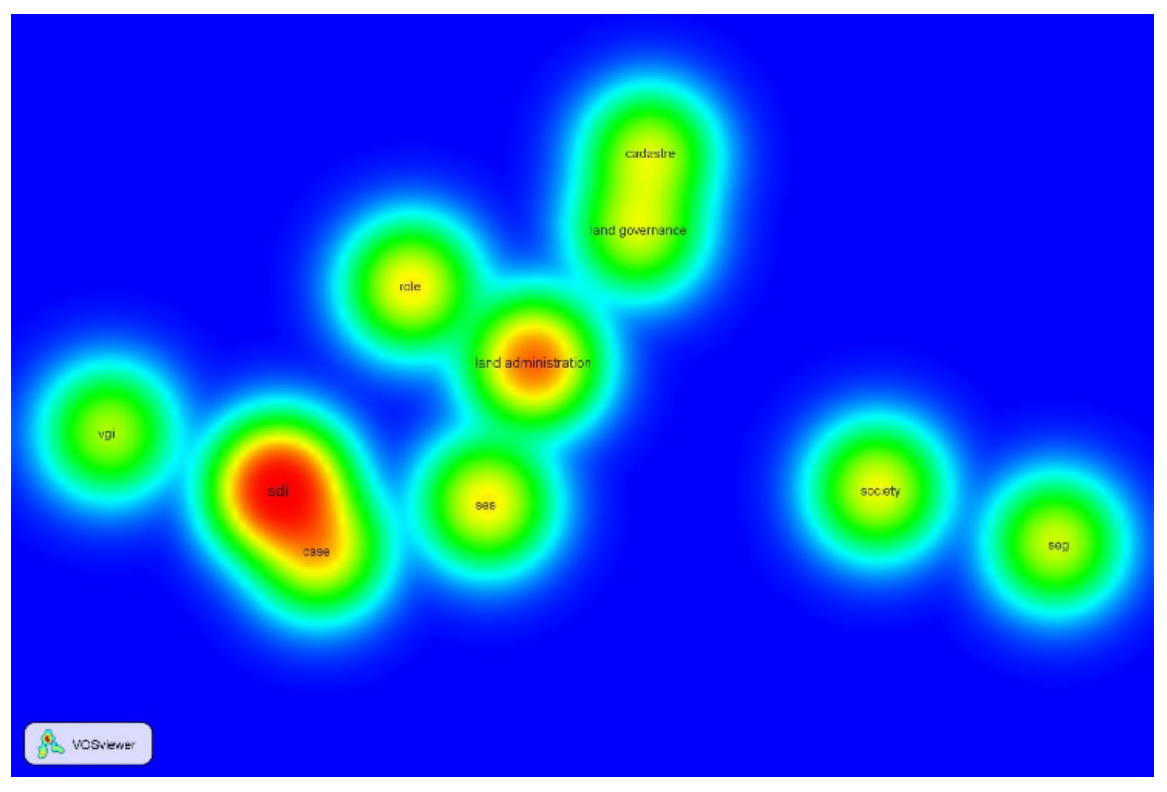

c) Sociedad capacitada geoespacialmente FUENTE: Elaboración del autor 
Durante este periodo hemos observado como la neogeografía está sujeta a una intensa controversia y debate (KEEN, 2007; ElWOOD, 2011; LESZCZYNSKI, 2014a; SUI, 2008; PARSONS, 2008; CARR, 2007). Revisar en profundidad esta controversia trasciende la pretensión de este artículo, pero se puede resumir en la polarización de dos posturas: una entusiasta y otra crítica.

Desde la perspectiva entusiasta se presenta a la neogeografía como un fenómeno que muestra la potencia de la inteligencia geográfica de las multitudes, una inteligencia colectiva que es una oportunidad de desarrollo económico y de empoderamiento de la sociedad. De manera especial sobresale el valor del prosumidor como experto de la realidad local (HARDY et al., 2012), en concreto, en su espacio de actividad de familiaridad cotidiana (GOODCHILD, 2009a). Además se subrayan las ventajas del capital humano que es capaz de movilizar la neogeografía frente al modelo productivo tradicional. Se destaca la actualización de datos en situaciones de crisis o emergencia, donde la velocidad y número de actualizaciones es superior al esquema tradicional (JACKSON et al., 2013).

El punto de vista crítico cuestiona el culto a lo amateur (KEEN, 2007) que impregna la sociedad, y el verdadero valor que poseen las contribuciones neogeográficas, especialmente por la falta de credibilidad y calidad, frente a las realizadas por los profesionales. En esta línea GRIRA et al., (2010) enfatizan que son la competencia y los conocimientos los factores que diferencian al experto del aficionado. La perspectiva crítica rechaza la ausencia de diseño cartográfico que caracteriza a la neogeografía. Además HAKLAY (2013) recalca el fracaso en la difusión de los valores democráticos y de empoderamiento que prometía la neogeografía. Este punto de vista concluye que el valor de la neogeografía queda reducido a un voluntariado de información geográfica, que recurre a los ciudadanos como sensores que pueden realizar contribuciones, valiosas en su justa medida, pero que rechaza la capacidad de análisis de los prosumidores.

\section{VI.3.3. La capacitación espacial de la sociedad}

En este candente debate sobre la neogeografía y el voluntariado de información geográfica se comienza a buscar y explorar la forma y los mecanismos de enriquecer los conjuntos de datos con la inclusión de las aportaciones de voluntarios y neogeógrafos mediante métodos que evalúen la credibilidad y calidad de los datos (FlANAGIN y METZGER, 2008; ESMAILI et al., 2013; POSER y DRANSCH, 2009). Se plantea el concepto de sociedad con capacidades, competencia o habilidades espaciales (WILLIAMSON et al., 2007). Esta perspectiva ofrece una clausura del debate neogeográfico y del voluntariado versus profesional. Este punto de vista es reciente y comienza a ser explorado 
(WILLIAMSON et al., 2011). Entre sus primeras aportaciones destaca la necesidad de realfabetizar geográficamente a la sociedad, con el fin de ayudar a la personas a usar, entender y utilizar los mapas en la toma decisiones (BUCKINHAM y DENNIS, 2009).

\section{LA SOCIEDAD: LA FINALIDAD DE LA PRODUCCIÓN Y DEL CONSUMO}

\section{VII.1. La producción}

Para qué producimos datos es una cuestión que antes se respondía desde el prisma de las organizaciones que disponían de una $S D I$. Hoy en día el foco de los estudios se ha centrado en cuál es la motivación de los neogeógrafos y los voluntarios para realizar contribuciones fuera del mercado laboral (COLEMAN et al., 2009; BUDHATHOKI y HAANDTHORNTHWAITE, 2013; LIU y PALEN, 2010).

También comienza a recibir atención el éxito de la estrategia empresarial desplegada por nuevas empresas que ofrecen servicios web 2.0. basados en la localización y los geoservicios, cuya táctica se basa en la socialización de los medios de producción espacial, los cuales despolitizan. Esta estrategia es disruptiva y sustenta un modelo de beneficio económico cuyo coste no se repercute al usuario del dato. Además, esta estrategia se exime de la responsabilidad por los efectos sociales del uso de estas tecnologías (LESZCZYNSKI, 2014a).

Los paradigmas que satisfacen mayor número de fenómenos son el Agile y el Suistanable (CUADRO 4).

\section{VII.2. EI consumo}

La irrupción de las tecnologías de la información espacial ha desplegado una agencia o capacidad de actuar en el mundo real que es novedosa. Según WILLIANSON et al. (2011) la agencia más destacada es la gestión y la organización de la economía, las personas y el medio ambiente. Otros autores exploran otras agencias: el empoderamiento de los grupos sociales, la participación ciudadana, la ciencia ciudadana (DODGE y KITCHIN, 2013; CONNORS et al., 2011), la visualización social, el ocio, la organización de actividades cotidianas, la gestión de las ciudades inteligentes, el geodiseño, los modelos $B I M$, o la visibilidad de grupos sociales entre otras.

Los paradigmas que satisfacen mayor número de fenómenos son el Cloud y el $\mathrm{Web} 2.0$ (CUADRO 4). 


\section{LA SOCIEDAD: LOS EFECTOS DE LA VÍA ECLÉCTICA}

El intento de satisfacer la demanda del consumidor actual sobre los datos espaciales ha dado origen a varios efectos en la producción y en el consumo, algunos de ellos no exentos de controversias.

El paradigma que satisface mayor número de fenómenos es el Agile (CUADRO 4).

\section{VIII.1. En la producción}

\section{VIII.1.a. Los neoterritorios}

El fenómeno inclusivo del dato espacial en la sociedad y el omnivorismo cultural de su consumo ha desencadenado la producción y el análisis de datos espaciales referidos que hasta ahora no habían recibido atención. A estos hechos geográficos se les ha definido como neoterritorios (DEL Río, 2012b). Se les denomina como neos porque suponen un re-descubrimiento de nuevas tierras ignotas sobre una geografía física ya descrita. Entre ellos destacan los nuevos paisajes humanos (FISCHER, 2008) o la cartografía de redes sociales (STEFANIDIS et al., 2013). Los neoterritorios no sólo han extendido el campo de aplicación de los datos espaciales; también han servido como canal para popularizarlos debido a la gran difusión que tienen en los medios de comunicación.

\section{VIII.1.b. La invisibilidad del mapa}

A pesar de la mayor disponibilidad y accesibilidad de la información que posibilita Internet, la economía de la atención que rige en ella hace que gran parte de los datos permanezcan invisibles. Esta paradoja de los mapas invisibles (DEL Río, 2011) demuestra la necesidad de mecanismos que faciliten la visibilidad del contenido. Entre esos sistemas destacan los metadatos pero son insuficientes sin la labor de otros agentes de intermediación que faciliten la difusión del dato en un canal saturado de información. Para solucionarlo se busca la optimización de contenidos que sean correctamente indexados en los buscadores en internet. A su vez, la economía de la atracción favorece la creación de sistemas de reputación y confiablidad.

VIII.1.c. El diseño cartográfico

El software de los sistemas de información geográfica $(G I S)$ y por extensión los relacionados con las tecnologías de información espacial, han cobrado un gran protagonismo como elemento de mediación (LESZCZYNSKI, 2014b). El GIS es simultáneamente un bien de producción y un medio de comunicación (SUI y GOODCHILD, 2003). 
El diseño cartográfico se ha revitalizado (FIELD y DEMAJ, 2012) a pesar de los anuncios de la muerte de los mapas (WoOD, 2003), de la amenaza de la pérdida de identidad de la cartografía (WOLODTSCHENKO, 2011), o la proliferación de los mapas clonados (DEL Río, 2009). Los tópicos que actualmente se están explorando son el diseño adaptado al tipo de usuario (KRAAK, 2011) o al canal, así como manifiestos para repensar la representación geográfica (ALTON et al., 2014).

\section{VIII.1.d. La producción esbelta}

El modelo ecléctico hace referencia a la conversión de los datos en valor, ya sea de uso o de cambio. La finalidad de la producción es el consumo. Este principio persigue la incorporación de los principios de trabajo de la producción esbelta (lean manufacturing) al proceso de creación de datos espaciales mediante el «Lean mapping» (DEL Río, 2012b).

VIII.1.e. El mercado de las bases de datos espaciales

Chilton (2009) plantea dos escenarios: ¿serán las empresas establecidas las que aprovechen el contenido generado por el usuario (UGC) para aumentar sus datos?, o ¿serán los nuevos competidores, que comercializan el $U G C$, los que utilicen estos datos para competir con los sistemas comerciales establecidos? Ante esta pugna el autor afirma que los verdaderos ganadores serán los consumidores, que tendrán disponibles grandes cantidades de datos geográficos precisos y actualizados y vaticina que los proveedores de datos se verán obligados a reconsiderar sus políticas de datos y modelos de precios.

\section{VIII.1.f. La equifinalidad en los datos espaciales}

El mercado de las bases de datos espaciales tradicionales se enfrenta a una ruptura de monopolios y a un proceso de equifinalidad espacial. Esto ha provocado que las bases de datos estén sometidas a un examen de calidad y satisfacción por parte del consumidor. CHILTON (2009) lo describe como una era de duelo entre bases de datos. Los casos en los que se ha detectado este proceso son los mapas bases y los relacionados con las situaciones de crisis. Esta superposición puede acabar en una alternativa o en un complemento (COLEMAN, 2013).

\section{VIII.1.g. Intermediarios}

El omnivorismo espacial de nuestra realidad ha provocado la necesidad de agentes y sistemas intermediarios que faciliten el acceso, reduciendo los riesgos y maximizando la confianza en el consumo. 


\section{VIII.2. En el consumo}

\section{VIII.2.a. La asimetría del consumo}

El consumo personal, científico, gubernamental y empresarial del dato está incorporando progresivamente la variable espacial en la toma de decisiones como medio estratégico de incremento de valor de un producto o servicio. Este alistamiento se está realizando mediante la integración de muchas fuentes de información.

Sin embargo, esta agregación es heterogénea y no está exenta de brechas. Por un lado se enuncia la brecha digital que trata sobre las posibilidades de acceso a Internet: el coste y la calidad de conexión en el entorno rural y urbano, por género, por edad o según el grado de desarrollo del país. Esta brecha, según SUI et al. (2013), plantea la muerte de la distancia y la importancia de la localización en el acceso a la información. Otros mecanismos de exclusión no tienen que ver con el acceso sino con el conocimiento. Un caso destacado es el generado por la posesión de competencias en programación que crea dos grupos: los que pueden crear apps y los que sólo pueden usarlas (ELWOOD, 2010). También se enuncia el Gap GIS que trata sobre la brecha en la adopción, accesibilidad, competencias y la trasferencia tecnológica en materia de datos espaciales en las organizaciones (DEL Río, 2012a).

VIII.2.b. La socialización, normalización y visibilidad de la geografía

El uso del dato espacial pasa a ser práctica cotidiana de la sociedad que lo utiliza en variadas estrategias de consumo; este consumo contribuye a difundir entre la sociedad algunos conceptos y tecnologías. A su vez, la web 2.0 proporciona un vehículo de normalización y acercamiento de las tareas de producción a la sociedad. Estos procesos proporcionan a la ciencia de la geografía diversas posibilidades para lograr una mayor visibilidad ante la sociedad.

\section{VIII.2.c. La hibridación entre el dato-virtual y el terreno-real}

El ciberespacio es la representación de los lugares físicos en Internet (GRAHAM y ZOOK, 2011). Entre el ciberespacio y el espacio real se producen relaciones. El dato espacial es la fuerza motriz que transfiere un sentido al lugar (FISHER, 2008), pero también se observa cómo los lugares se interrelacionan con el dato virtual en los digiplace (ZOOK y GRAHAM, 2007) o el placelooging (KOTTAMASU, 2007).

Ante esta comunicación bidireccional entre la virtualidad del dato y el terreno se plantea el concepto de espacio hibridado (DE SOUZA E SILVA, 2006) y la permeabilidad entre el modelo y la realidad (FARMAN, 2012). Se explora el concepto de localidades en red como forma de clausura (DE SoUZA E 
SILVA y FRITH, 2010) y se discute sobre la producción automática del dato (THRIFT y FRENCH, 2002) y la integración y convergencia con otros sistemas de información (DE MAN, 2007).

\section{CONCLUSIONES}

Esta revisión se ha centrado en inventariar los cambios en la producción y consumo como hilo conductor para narrar los fenómenos que se han producido alrededor de los datos espaciales. A partir de la revisión se ha propuesto una vía ecléctica para describir y explicar el modelo actual de consumo y producción de datos espaciales. La vía ecléctica de producción y consumo se basa en la búsqueda del valor del dato espacial, el papel de las geocomunidades y los roles de los prosumidores en la sociedad. Además, la vía ecléctica ofrece una explicación a la superposición de modos de producción y consumo. Estos modos están muy ligados a un nivel muy simple de geograficidad vinculado con la localización del elemento sobre el territorio.

La metodología utilizada para organizar la descripción de los fenómenos observados ofrece un marco de interés para dibujar un mapa del momento actual. Este mapa permite navegar entre los profundos cambios que estamos observando, muchos de los cuales requieren de mayor estudio científico. El proceso analítico jerárquico tiene además la utilidad de orientar la gestión de geocomunidades y enmarcar la producción e implantación de sistemas de información basados en datos especiales desde los paradigmas de producción. La adaptación del árbol jerárquico a cada situación concreta permite ajustar este marco conceptual a cada sector geoespacial concreto y determinar que paradigma es el más conveniente para orientar la producción en cada caso.

El análisis bibliográfico muestra, durante el periodo revisado, un gran interés en el fenómeno neogeográfico, sin que existan trabajos que extiendan el análisis al conjunto del fenómeno de producción y consumo de los datos espaciales que están concurriendo en el contexto actual. La revisión detecta la existencia de brechas que son interesantes áreas de trabajo y estudio, entre las que destacan el análisis geográfico, el diseño cartográfico, la gestión de las geocomunidades y la capacitación de la sociedad.

\section{AGRADECIMIENTOS}

El autor quisiera agradecer la amable invitación de D. José Cortizo Álvarez a participar en este número especial y las sugerencias de los revisores, cuyas aportaciones han mejorado la calidad de este artículo. 


\section{BIBLIOGRAFÍA}

Alton, C.; Ayub, Z.; Chen, A.; Estrada, L.; Kollar, J.; LeOnard, P.; Pavlinic, M.; ViglaKIS, A. y WiLSON, M. 06/12/2014: «Thinking/Making Geographic Representation» en Critical GIS.com [Mensaje en un blog].

http:/criticalgis.blogspot.com.es/2014/06/thinkingmaking-geographic-representation.html

AnTHONY, D.; SEAN, W. S. y Williamson, T. (2007): «The Quality of Open Source Production: Zealots and Good Samaritans» en the Case of Wikipedi; (D. C. S. T. R. TR2007-606 dirs.). Dartmouth College Computer Science. Hanover, NH, USA.

AgArWAL, A.; SHANKAR, R. y TIWARI, M. (2006): «Modeling the metrics of lean, agile and leagile supply chain: An ANP-based approach». European Journal of Operational Research, $\mathrm{n}^{\mathrm{o}}$ 173(1), 211-225.

AgArwal, A.; SHANKAR, R. y TIWARI, M. (2007): «Modeling agility of supply chain». Industrial marketing management, $\mathrm{n}^{\mathrm{o}} 36(4), 443-457$.

ARIZA, F. J. (2012): «La cartografía continua: de lo global a lo local, un espacio sin cortes y de múltiples aplicaciones», Ponencia en La globalización de la cartografia. V encuentro del grupo de trabajo de cartotecas públicas hispano-lusas (Santander, 5 de octubre de 2012). Universidad de Cantabria.

AtzMAnstorfer, K. y BlaschKe, T. (2013): «The Geospatial Web: A Tool to Support the Empowerment of Citizens through E-Participation?», en Citizen EParticipation in Urban Governance: Crowdsourcing and Collaborative Creativity (NuNES,C.S. dirs.). Hershey, PA, USA, IGI Global, 1-392.

BAuman, Z. (2000): Liquid Modernity. UK, Cambridge: Polity.

BECK, H. (2005): Medienökonomie: Print, Fernsehen und Multimedia. Springer-Verlag Berlin Heidelberg.

BERDOU, E. (2012): «Participatory Technologies and Participatory Methodologies: Ways Forward for Innovative Thinking and Practice», en IKM working paper, vol. 17 (CUMmINGS, T. dirs.), Bonn, Germany.

BI, Z. (2011): «Revisiting system paradigms from the viewpoint of manufacturing sustainability». Sustainability, no 3(9), 1323-1340.

BI, Z.; DA XU, L. y WANG, C. (2014): «Internet of Things for enterprise systems of modern manufacturing». Industrial Informatics, IEEE Transactions on, $\mathrm{n}^{\circ} 10(2)$, 1537-1546.

BouRdiEU, P. (2002): «El mercado lingüístico» en Sociología y cultura. México, Grijalbo, Conaculta, 143-158.

BRUNS, A. (2008): «The future is user-led: The path towards widespread produsage». Fibreculture Journal, $\mathrm{n}^{\mathrm{o}} 11$.

BUCKINGHAM, W. R. y DENNIS JR, S. F. (2009): «Cartographies of Participation: How the changing natures of cartography has opened community and cartographer collaboration». Cartographic perspectives, $\mathrm{n}^{\circ}$ (64), 55-61.

BudHATHOKI, N. R.; BRUCE, B. y NedOVIC-Budic, Z. (2008): «Reconceptualizing the role of the user of spatial data infrastructure». GeoJournal, n ${ }^{\circ}$ 72(3-4), 149160 . 
BudhathoKi, N. R. y HAYTHORnTHWAite, C. (2013): «Motivation for Open Collaboration: Crowd and Community Models and the Case of OpenStreetMap». American Behavioral Scientist, $\mathrm{n}^{\circ}$ 57(5), 548-575.

CAPEL, H. (2012): «Nuevas geografías y neogeografías» en Filosofía y ciencia en la geografía contemporánea. Una introducción a la Geografía. Barcelona, Ediciones del Serbal, 411-439.

CARVAlHO, H. y CRUZ-Machado, V. (2011): Integrating Lean, Agile, Resilience and Green Paradigms in Supply Chain Management (LARG_SCM), INTECH Open Access Publisher.

CARDONA, G. y SANZ, J. P. (2015): «Publication analysis of the contact lens field: What are the current topics of interest?». Journal of Optometry, $\mathrm{n}^{\circ}$ 08(01), 33-39.

CARR, N. G. (2007): «The Ignorance of Crowds The open source model can play an important role in innovation, but know its limitations». Strategy and Business, ${ }^{\circ}$ 47.

CARTWRIGHT, W. (2012): «Neocartography: Opportunities, Issues and Prospects». South African Journal of Geomatics, $\mathrm{n}^{\circ}$ 1(1), 14-31.

CASTELlS, M. (2004): «1. Informacionalismo, redes y sociedad red: una propuesta teórica» en La sociedad red: una visión global (CASTELLS, M. dir.). Alianza Editorial, $27-78$.

CHILTON, S. (2009): «Crowdsourcing is radically changing the geodata landscape: case study of OpenStreetMap», Ponencia en Proceedings of the 24th International Cartographic Conference (Santiago de Chile, 15-21 November 2009).

CHRISTOPHER, M. (2000): «The agile supply chain: competing in volatile markets». Industrial marketing management, $\mathrm{n}^{\mathrm{o}}$ 29(1), 37-44.

Christopher, M. y PECK, H. (2004): «Building the resilient supply chain». The International Journal of Logistics Management, $\mathrm{n}^{\mathrm{o}}$ 15(2), 1-14.

CHRISTOPHER, M. y TOWILL, D. R. (2000): «Supply chain migration from lean and functional to agile and customised». Supply Chain Management: An International Journal, no 5(4), 206-213.

Christopher, M. y TOWILL, D. (2001): «An integrated model for the design of agile supply chains». International Journal of Physical Distribution \& Logistics Management, $\mathrm{n}^{\circ} 31(4), 235-246$.

ClarKe, K. C. (2013): «What is the World's Oldest Map?». The Cartographic Journal, $\mathrm{n}^{\circ}$ 50(2), 136-143.

Coleman, D. J. (2013): «Potential contributions and challenges of VGI for conventional topographic base-mapping programs» en Crowdsourcing Geographic Knowledge (SuI, D.; ElWOOD, S. y GoOdCHILD, M. dirs.). Springer Netherlands, 245-26.

Coleman, D. J.; Georgiadou, Y. y Labonte, J. (2009): «Volunteered geographic information: The nature and motivation of producers». International Journal of Spatial Data Infrastructures Research, $\mathrm{n}^{\circ}$ 4(1), 332-358.

CONNORS, J. P.; LEI, S. y Kelly, M. (2011): «Citizen Science in the Age of Neogeography: Utilizing Volunteered Geographic Information for Environmental Monitoring». Annals of the Association of American Geographers, $\mathrm{n}^{\circ}$ 102(6), 12671289. 
Cooper, A. K.; Coetzee, S.; KaczmareK, I.; Kourie, D. G.; IwaniaK, A. y KubiK, T. (2011): "Challenges for Quality in Volunteered Geographical Information», Ponencia en Proceedings of the AfricaGEO 2011 Conference (Cape Town, South Africa, 31 de mayo a 2 de junio de 2011).

CRAmpton, J. W. (2009): «Cartography: maps 2.0». Progress in Human Geography, $n^{\circ} 33(1), 91-100$.

CREIGHTON, J. L. (2005): The public participation handbook: making better decisions through citizen involvement. San Francisco, USA, Jossey-Bass.

DAVENPORT, T. y BECK, J. (2002): The Attention Economy: Understanding the New. Currency of Business, Harvard: Harvard Business Press.

DEIF, A. M. (2011): «A system model for green manufacturing». Journal of Cleaner Production, $\mathrm{n}^{\mathrm{o}}$ 19(14), 1553-1559.

DE MAN, W. H. E. (2007): «Are Spatial Data Infrastructures Special?» en Research and Theory in Advancing Spatial Data Infrastructure Concepts (ONSRUD, H. dirs.). Redlands, CA, ESRI Press, 33-54.

De SouzA E Silva, A. (2006): «From Cyber to Hybrid Mobile Technologies as Interfaces of Hybrid Spaces». Space and culture, $n^{\circ}$ 9(3), 261-278.

De SouzA E Silva, A. y FriTH, J. (2010): «Locative Mobile Social Networks: Mapping Communication and Location in Urban Spaces». Mobilities, $\mathrm{n}^{\circ}$ 5(4), 485-505.

DEL RÍO, J. 10/03/2009: «El mundo feliz de los mapas clonados» en Orbemapa.com [Mensaje en un blog].

http://www.orbemapa.com/2009/03/el-mundo-feliz-de-los-mapas-clonados.html

DEL Río, J. (2011): Mapas invisibles. España, Bubook.

DEL Río, J. 17/05/2012a: «La brecha digital en la adopción de la tecnología SIG» en Orbemapa.com [Mensaje en un blog]. http://www.orbemapa.com/2012/05/brecha-digital-sig.html

DEL Río, J. 11/09/2012b: «El consumo de datos espaciales es la finalidad de la producción cartográfica», en Orbemapa.com [Mensaje en un blog]. http://www.orbemapa.com/2012/09/el-consumo-de-datos-espaciales-es-la-finalidad-de-la-produccion-cartografica.html

DEL Río, J. 27/07/2012c: «Neoterritorios» en Orbemapa.com [Mensaje en un blog]. http://www.orbemapa.com/2012/07/neoterritorios.html

Dessers, E.; Crompvoets, J.; Vandenbroucke, D.; VAnCAuWenberghe, G.; JANSSEN, K.; VANHAVERBEKE, L. y HoOtegem, G. V. (2012): «A Multidisciplinary Research Framework for Analysing the Spatial Enablement of Public Sector Processes». International Journal of Spatial Data Infrastructures Research, $\mathrm{n}^{\mathrm{0}} 7$, 125-150.

DodGE, M. y KITCHIN, R. (2013): «Crowdsourced cartography: mapping experience and knowledge». Environment and Planning A, no 45(1), 19-36.

Dodge, M.; Perkins, C. y KitChin, R. (2009): «12. Mapping modes, methods and moments: a manifesto for map studies» en Rethinking Maps (ROUTLEDGE, dirs.). Vol. 28. New York, Routledge, 1ed., 220-243.

DUBEY, R. y GUNASEKARAN, A. (2015): «Agile manufacturing: framework and its empirical validation». The International Journal of Advanced Manufacturing Technology, $\mathrm{n}^{\mathrm{o}}$ 76(9-12), 2147-2157. 
EDNEY, M. H. (2005): «Putting "Cartography" into the History of Cartography: Arthur H. Robinson, David Woodward, and the Creation of a Discipline». Cartographic perspectives, $\mathrm{n}^{\circ}$ 51, 14-29.

EDNEY, M. H. (2011): «Reflection Essay: Progress and the Nature of Cartography» en Classics in Cartography: Reflections on Influential Articles from Cartographica (DODGE, M. dir.). Chichester, UK, John Wiley \& Sons, 331-342.

ELMARAGHY, H. A. (2005): «Flexible and reconfigurable manufacturing systems paradigms». International journal of flexible manufacturing systems, $\mathrm{n}^{\circ} 17(4), 261-$ 276.

ELWOOD, S. (2010): «Geographic information science: emerging research on the societal implications of the geospatial web». Progress in Human Geography, $\mathrm{n}^{\mathrm{o}}$ 34(3), 349-357.

ELWOOD, S. (2011): «Geographic Information Science: Visualization, visual methods, and the geoweb». Progress in Human Geography, no 35(3), 401-408.

ELWOOD, S. y LESZCZYNSKI, A. (2011): «Privacy, reconsidered: New representations, data practices, and the geoweb». Geoforum, $\mathrm{n}^{\circ}$ 42(1), 6-15.

ELWOOD, S. y LESZCZYNSKI, A. (2013): «New spatial media, new knowledge politics». Transactions of the Institute of British Geographers, $\mathrm{n}^{\circ}$ 38(4), 544-559.

ESMAILI, R.; NASERI, F. y ESMAILI, A. (2013): «Quality Assessment of Volunteered Geographic Information». American Journal of Geographic Information System, $\mathrm{n}^{\circ} 2(2), 19-26$.

FARMAN, J. (2012): The Mobile Interface of Everyday Life: Embodied Space and Locative Media, Routledge.

FIELD, K. y DEMAJ, D. (2012): «Reasserting design relevance in cartography: some concepts». The Cartographic Journal, no 49(1), 70-76.

FISCHER, F. (2008): «Implications of the usage of mobile collaborative mapping systems for the sense of place», Ponencia en Real Corp 08 (Vienna, Austria, 19 a 21 de mayo de 2008). corp.at, 583-587.

FLANAGIN, A. J. y METZGER, M. J. (2008): «The credibility of volunteered geographic information». GeoJournal, n ${ }^{\circ}$ 72(3-4), 137-148.

Gartner, G., Bennett, D. A. y MORITA, T. (2007): «Towards Ubiquitous Cartography». Cartography and Geographic Information Science, $\mathrm{n}^{\circ}$ 34(4), 247-257.

GeOrgiadou, Y.; HARVEY, F. y Miscione, G. (2007): A bigger picture: information systems and spatial data infrastructure research perspectives. En Proceedings of the 10th AGILE international conference on geographic information science: the European Information Society: leading the way with geoinformation, Aalborg, Denmarkdirs.), 8-11.

GIFF, G. A. y CROMPVOETS, J. (2008): «Performance indicators a tool to support spatial data infrastructure assessment». Computers, Environment and Urban Systems, $\mathrm{n}^{\mathrm{o}} 32(5), 365-376$.

Goldsby, T. J.; GrIfFIS, S. E. y RoATH, A. S. (2006): «Modeling lean, agile, and leagile supply chain strategies». Journal of business logistics, $\mathrm{n}^{\circ} 27(1), 57-80$.

GOODCHILD, M.F. (2007): «Citizens as sensors: the world of volunteered geography». GeoJournal, no 69(4), 211-221.

GoODCHILD, M.F. (2009a): «NeoGeography and the nature of geographic expertise». Journal of Location Based Services, n 3(2), 82-96. 
GOODCHILD, M.F. (2009b): «Geographic information systems and science: today and tomorrow». Annals of GIS, $\mathrm{n}^{\circ} 15(1), 3-9$.

Gorman, S. (16/09/2009): «Huffman's Three Principles for Data Sharing Off the Map » en Official Blog of FortiusOne [Mensaje en un blog]. http://blog.geoiq.com/2009/09/16/huffman

GRAHAM, M. (2011): «Time machines and virtual portals. The spatialities of the digital divide». Progress in Development Studies, ${ }^{\circ}$ 11(3), 221-227.

GrAHAM, M. y ZOOK, M. (2011): «Visualizing Global Cyberscapes: Mapping UserGenerated Placemarks». Journal of Urban Technology, $\mathrm{n}^{\circ}$ 18(1), 115-132.

GRIRA, J.; BÉDARD, Y. y ROCHE, S. (2010): «Spatial data uncertainty in the VGI world: Going from consumer to producer». Geomatica, $\mathrm{n}^{\circ}$ 64(1), 61-72.

GUNASEKARAN, A. y YUSUF, Y. (2002): «Agile manufacturing: a taxonomy of strategic and technological imperatives». International Journal of Production Research, $\mathrm{n}^{\circ}$ 40(6), 1357-1385.

GuPTILL, S. C. (2007): «GIScience, the NSDI, and GeoWikis». Cartography and Geographic Information Science, $\mathrm{n}^{\mathrm{o}}$ 34(2), 165-166.

HAKLAY, M. (2012): «Geographic information science: tribe, badge and sub-discipline». Transactions of the Institute of British Geographers, $n^{\circ} 37(4), 477-481$.

HAKLAY, M. (2013): «Neogeography and the delusion of democratisation». Environment and Planning $A, \mathrm{n}^{\circ}$ 45(1), 55-69.

Haklay, M.; Singleton, A. y PARKer, C. (2008): «Web Mapping 2.0: The Neogeography of the GeoWeb». Geography Compass, n 2(6), 2011-2039.

HARDY, D.; FREW, J. y GOODCHILD, M. F. (2012): «Volunteered geographic information production as a spatial process». International Journal of Geographical Information Science, $\mathrm{n}^{\mathrm{o}} 26(7), 1191-1212$.

HARLEY, J. B. (1990): «Introduction: Text and Contexts in the Interpretation of Early Maps» en From Sea Charts to Satellite Images: Interpreting North American History through Maps (D. BUISSERET, dir.). Chicago, 3-15.

HARZING, A.-W. y VAN DER WAL, R. (2007): «Google Scholar: the democratization of citation analysis». Ethics in science and environmental politics, $\mathrm{n}^{\circ} 8(1), 61-73$.

HEIPKE, C. (2010): «Crowdsourcing geospatial data». ISPRS Journal of Photogrammetry and Remote Sensing, ${ }^{\circ}$ 65(6), 550-557.

HINES, P.; HolWEG, M. y RICH, N. (2004): «Learning to evolve: A review of contemporary lean thinking». International Journal of Operations \& Production Management, $\mathrm{n}^{\circ} 24(10), 994-1011$.

HOEKSTRA, S. y ROMME, J. (1992): Integral logistic structures: developing customeroriented goods flow. London, Reino Unido, McGraw-Hill, London.

Jackson, S. P.; Mullen, W.; Agouris, P.; Crooks, A.; Croitoru, A. y STEFANIDIS, A. (2013): «Assessing completeness and spatial error of features in volunteered geographic information». ISPRS International Journal of Geo-Information, $\mathrm{n}^{\mathrm{o}} 2(2), 507-530$.

JANSSEN, K. (2011): «A legal approach to assessing Spatial Data Infrastructures» en A Multiview framework to assess spatial data infraestructures (CROMPVOETS, J.; RAJABIFARD, A.; VAn LoenenYt, B. y Delgado Fernández, T. dirs.). Melbourne, Australia Space for Geo-Information (RGI), Wageningen, University and 
Centre for SDIs and Land Administration y Department of Geomatics, The University of Melbourn, Chapter 13, 255-272.

KARROW, R. (2007): «Centers of Map Publishing in Europe, 1472-1600» en Cartography in the European Renaissance (WOODWARD, D. dir.), vol. 3. Chicago, IL, The University of Chicago Press, 611-621.

KEEN, A. (2007): The cult of the amateur: How today's internet is killing our culture. New York, USA, Doubleday/Currency.

KORPI, J. y AHONEN-RAINIO, P. (2010): "Cultural constraints in the design of pictographic symbols». The Cartographic Journal, no 47(4), 351-359.

Kottamasu, R. (2007): Placelogging: Mobile spatial annotation and its potential use to urban planners and designers, Massachusetts Institute of Technology.

KRAAK, M.-J. (2011): «Is there a need for neo-cartography?» Cartography and Geographic Information Science, $\mathrm{n}^{\mathrm{o}}$ 38(2), 73-78.

KURWAKUMIRE, E. (2014): «Towards a Public Sector GIS Evaluation Methodology». South African Journal of Geomatics, $\mathrm{n}^{\mathrm{o}} 3(1), 33-52$.

LAU, K. H. (2011): «Benchmarking green logistics performance with a composite index». Benchmarking: An International Journal, $\mathrm{n}^{\circ}$ 18(6), 873-896.

LEE, C. I. S. G.; FELPS, W. y BARUCH, Y. (2014): «Toward a taxonomy of career studies through bibliometric visualization». Journal of Vocational Behavior, $\mathrm{n}^{\mathrm{o}}$ 85(3), 339-351.

LESZCZYNSKI, A. (2014a): «On the Neo in Neogeography». Annals of the Association of American Geographers, $\mathrm{n}^{\circ}$ 104(1), 60-79.

LESZCZYNSKI, A. (2014b): «Spatial Media/tion». Progress in Human Geography, ${ }^{\circ}$ Published online before print.

LIU, S. B. y PALEN, L. (2010): «The New Cartographers: Crisis Map Mashups and the Emergence of Neogeographic Practice». Cartography and Geographic Information Science, $\mathrm{n}^{\mathrm{o}}$ 37(1), 69-90.

MEHO, L. I. y YANG, K. (2007): «Impact of data sources on citation counts and rankings of LIS faculty: Web of Science versus Scopus and Google Scholar». Journal of the American society for information science and technology, $\mathrm{n}^{\circ} 58(13), 2105$ 2125 .

Maciá Pérez, F.; Berná Martínez, J. V.; Marcos Jorquera, D.; Lorenzo FONSECA, I. y FERRÁNDIZ COLMEIRO, A. (2012): «A new paradigm: cloud agile manufacturing». International Journal of Advanced Science and Technology. Vol. 45 (Aug. 2012), 47-54

MASON-JONES, R.; NAYLOR, B. y TOWILL, D. R. (2000): «Lean, agile or leagile? Matching your supply chain to the marketplace». International Journal of Production Research, $\mathrm{n}^{\mathrm{0}}$ 38(17), 4061-4070.

MeHrABI, M. G.; Ulsoy, A. G. y KOREN, Y. (2000): «Reconfigurable manufacturing systems: key to future manufacturing». Journal of Intelligent manufacturing, $\mathrm{n}^{\mathrm{o}}$ 11(4), 403-419.

MELton, T. (2005): «The benefits of lean manufacturing: what lean thinking has to offer the process industries». Chemical Engineering Research and Design, $\mathrm{n}^{\circ}$ 83(6), 662-673. 
MorerA, C. O.E., C.; REY, D. I. y GuIMET, J. (2012): «Evaluación de una IDE desde su caracterización hasta su impacto en la sociedad» en Fundamentos de las Infraestructuras de Datos Espaciales (IDE) (M. Á. Bernabé-Poveda y C. M. LópezVázquez, dirs.). Madrid, España, UPM Press,

Moseme, M. T. y VAn ElZAKKeR, C. (2012): «Neogeography Map Users and Uses», Ponencia en Proceedings of AutoCarto (Columbus, Ohio, USA, 16-18 de Septiembre de 2012).

Muehlenhaus, I. (2013): «The design and composition of persuasive maps». Cartography and Geographic Information Science, $\mathrm{n}^{\circ}$ 40(5), 401-414.

Muenlenhaus, I. (2014): «Going Viral: The Look of Online Persuasive Maps». Cartographica: The International Journal for Geographic Information and Geovisualization, $\mathrm{n}^{\mathrm{o}}$ 49(1), 18-34.

NAYLOR, J. B.; NAIM, M. M. y BERRY, D. (1999): «Leagility: integrating the lean and agile manufacturing paradigms in the total supply chain». International Journal of production economics, $\mathrm{n}^{\circ}$ 62(1), 107-118.

NEIS, P. y ZIPF, A. (2012): «Analyzing the contributor activity of a volunteered geographic information project-The case of OpenStreetMap». ISPRS International Journal of Geo-Information, $\mathrm{n}^{\circ}$ 1(2), 146-165.

NEWMAN, M. Z. (2010): «New media, young audiences and discourses of attention: from Sesame Street to 'snack culture'». Media, Culture \& Society, n 32(4), 581596.

Noucher, M. y GOLAY, F. (2010): «From the Assessment of Spatial Data Infrastructure to the Assessment of Community of Practice: Advocating an Approach by Uses», Ponencia en GeoValue 2010: value of geoinformation (Hamburg, Germany, Septiembre 2010). 1,7.

O'BRIEN, C. (2013): «Fifty years of shifting paradigms». International Journal of Production Research, $\mathrm{n}^{\circ}$ 51(23-24), 6740-6745.

OlHager, J. (2012): «The Role of Decoupling Points in Value Chain Management» en Modelling Value (H. Jodlbauer, J. Olhager, R. J. Schonberger, dirs.). Physica-Verlag HD, 37-47.

O'REILLY, T. (2007): «What is Web 2.0: Design patterns and business models for the next generation of software». Communications \& strategies, $\mathrm{n}^{\circ}(1), 17$.

OrtegA, F. G. y BARAHONA, J. M. (2007): «Quantitative Analysis of the Wikipedia Community of Users», Ponencia en Proceedings of the WikiSym Conference (New York, USA, ACM Press, 75-86.

PARSONS, E. 01/09/2008: «Cartography is dead, long live the map makers», en edparsons [Mensaje en un blog].

http://www.edparsons.com/2008/09/cartography-is-dead-long-live-the-map-mak$\underline{\text { ers/ }}$

PECK, H. (2005): «Drivers of supply chain vulnerability: an integrated framework». International Journal of Physical Distribution \& Logistics Management, $\mathrm{n}^{\mathrm{o}} 35(4)$, 210-232.

PETERSON, R. A. (1992): «Understanding audience segmentation: From elite and mass to omnivore and univore». Poetics, $\mathrm{n}^{\circ}$ 21(4), 243-258.

Plewe, B. (2007): «Web cartography in the United States». Cartography and Geographic Information, $\mathrm{n}^{\mathrm{o}}$ 34(2), 133-136. 
POSER, K. y DRANSCH, D. (2010): «Volunteered geographic information for disaster management with application to rapid flood damage estimation». Geomatica, $\mathrm{n}^{\mathrm{o}}$ 64(1), 89-98.

Priedhorsky, R.; Chen, J.; LAm, S. K.; PAnciera, K.; Terveen, L. y Reidl, J. (2007): "Creating, Destroying, and Restoring Value in Wikipedia», Ponencia en International ACM Conference on Supporting Group Work (Sanibel Island, FL, 4-7 noviembre de 2007).

RANA, S. y JoLIVEAU, T. (2009): «NeoGeography: an extension of mainstream geography for everyone made by everyone?». Journal of Location Based Services, $\mathrm{n}^{\circ}$ $3(2), 75-81$.

Ritzer, G. y Jurgenson, N. (2010): «Production, Consumption, Prosumption The nature of capitalism in the age of the digital 'prosumer'». Journal of consumer culture, $\mathrm{n}^{\mathrm{o}} 10(1), 13-36$.

RAO, P. y HOLT, D. (2005): «Do green supply chains lead to competitiveness and economic performance?» International Journal of Operations \& Production Management, $\mathrm{n}^{\circ}$ 25(9), 898-916.

ROBERTS, K. (2005): Lovemarks: The future beyond brands, PowerHouse Books.

SAATY, T.L. (1980): The Analytic Hierarchy Process. McGraw-Hill, New York.

SHEPPARD, E. (2005): «Knowledge production through critical GIS: genealogy and prospects». Cartographica: The International Journal for Geographic Information and Geovisualization, $\mathrm{n}^{\circ}$ 40(4), 5-21.

Stefanidis, A.; Crooks, A. y RADZIKOWSKI, J. (2013): «Harvesting ambient geospatial information from social media feeds». GeoJournal, $\mathrm{n}^{\mathrm{o}}$ 78(2), 319-338.

Streilein, A. y Kellenberger, T. (2010): «Crowd Sourcing for Updating National Databases», Ponencia en Proceedings EuroSDR Workshop Berne (Wabern, Switzerland, 20-21 Agosto de 2009). EuroSDR Official Publication ( $\mathrm{n}^{\circ}$ 57).

SUI, D. Z. (2008): «The wikification of GIS and its consequences: Or Angelina Jolie's new tattoo and the future of GIS». Computers, Environment and Urban Systems, $n^{\circ} 32(1), 1-5$.

SUI, D. Z. y GOODCHILD, M. F. (2003): «A tetradic analysis of GIS and society using McLuhan's law of the media». Canadian Geographer / Le Géographe canadien, $\mathrm{n}^{\circ} 47(1), 5-17$.

SuI, D.; GoodchILD, M. y Elwood, S. (2013): «Volunteered Geographic Information, the Exaflood, and the Growing Digital Divide» en Crowdsourcing Geographic Knowledge (SUI, D.; ElWOOD, S.; y GOODCHILD, M. dirs.). Springer Netherlands, 1-12.

THRIFT, N. y FRENCH, S. (2002): «The automatic production of space». Transactions of the Institute of British Geographers, $\mathrm{n}^{\circ}$ 27(3), 309-335.

Tsou, M. H. (2011): «Revisiting Web Cartography in the United States: the Rise of User-Centered Design». Cartography and Geographic Information Science, $\mathrm{n}^{\circ}$ 38(3), 250-257.

TURNER, A. (2006): Introduction to neogeography, O'Reilly Media, Inc.

TURNER, A. y GORMAN, S. (2013): «Neogeography Interfaces for Emergent Communities», Ponencia en GeoHCI Workshop at CHI 2013 (April 27-28, 2013). Paris, France. 
Utrilla, P.; Mazo, C.; Sopena, M. C.; MartíneZ-BeA, M. y Domingo, R. (2009): "A palaeolithic map from 13,660 calBP: engraved stone blocks from the Late Magdalenian in Abauntz Cave (Navarra, Spain)». Journal of Human Evolution, $\mathrm{n}^{\circ}$ 57(2), 99-111.

VAN HOEK, R. I. (1998): «Reconfiguring the supply chain to implement postponed manufacturing». The International Journal of Logistics Management, $\mathrm{n}^{\mathrm{o}}$ 9(1), 95 110.

VIRRANTAUS, K.; FAIRBAIRN, D. y KRAAK, M.-J. (2009): «ICA research agenda on cartography and GIScience». Cartography and geographic information science, $n^{\circ} 36(2), 209-222$.

Vonderembse, M. A.; Uppal, M.; HuAnG, S. H. y Dismukes, J. P. (2006): «Designing supply chains: Towards theory development». International Journal of production economics, $\mathrm{n}^{\mathrm{o}}$ 100(2), 223-238.

WARF, B. y SUI, D. (2010): «From GIS to neogeography: ontological implications and theories of truth». Annals of GIS, $\mathrm{n}^{\mathrm{o}} 16(4), 197-209$.

WENGER, E. (1998): Communities of practice: Learning, meaning, and identity. Cambridge, UK, Cambridge University Press.

WESKE, M. (2012): Business process management: concepts, languages, architectures, Springer Science \& Business Media.

WILLIAMSON, I.; RAJABIFARD, A. y BINNS, A. (2007): «The role of spatial data infrastructures in establishing an enabling platform for decision making in Australia». Research and Theory in Advancing Spatial Data Infrastructure Concepts, $\mathrm{n}^{\circ}, 121$ 132.

Williamson, I.; Rajabifard, A.; Wallace, J. y BenNETT, R. (2011): «Spatially enabled society», Ponencia en FIG Working Week 2011. Bridging the Gap between Cultures (Marrakech, Morocco, 18-22 Mayo 2011).

WILSON, M. W. y GRAHAM, M. (2013): «Neogeography and volunteered geographic information: A conversation with Michael Goodchild and Andrew Turner». Environment and Planning $A, \mathrm{n}^{\circ} 45,10-18$.

WOLODTSCHENKO, A. (2011): "Quo vadis classic cartosemiotics \& quo vadis theoretical cartography?» Journal for Theoretical Cartography, $\mathrm{n}^{\circ}$ 4, 1-18.

WOMACK, J. P.; JONES, D. T. y RoOS, D. (1990): Machine that changed the world. New York, USA, Rawson Associates.

WooD, D. (2003): «Cartography is dead (thank God!)». Cartographic perspectives, $\mathrm{n}^{\mathrm{o}}(45), 4-7$.

XU, X. (2012): «From cloud computing to cloud manufacturing». Robotics and computer-integrated manufacturing, $\mathrm{n}^{\circ}$ 28(1), 75-86.

YAO, Q.; CHEN, K.; YAO, L.; LYU, P.-H.; YANG, T.-A.; LUO, F.; CHEN, S.-Q.; HE, L.Y. y LIU, Z.-Y. (2014): «Scientometric trends and knowledge maps of global health systems research». Health Research Policy and Systems, $\mathrm{n}^{\circ}$ 12(1), 26.

ZOOK, M. A. y GRAHAM, M. (2007): «The creative reconstruction of the Internet: Google and the privatization of cyberspace and DigiPlace». Geoforum, no 38(6), 1322-1343. 